\title{
Plin5/p-Plin5 Guards Diabetic CMECs by Regulating FFAs Metabolism Bidirectionally
}

\author{
Jin Du, ${ }^{1}$ Juanni Hou, ${ }^{2}$ Juan Feng, ${ }^{1}$ Hong Zhou, ${ }^{3}$ Heng Zhao, ${ }^{4}$ Dachun Yang ${ }^{D},{ }^{1}$ De Li, \\ Yongjian Yang $\mathbb{B}^{1},{ }^{1}$ and Haifeng Pei ${ }^{1}{ }^{1}$ \\ ${ }^{1}$ Department of Cardiology, The General Hospital of Western Theater Command, Chengdu 610083, China \\ ${ }^{2}$ Department of Gastroenterology, The General Hospital of Western Theater Command, Chengdu 610083, China \\ ${ }^{3}$ Department of Respiration, The General Hospital of Western Theater Command, Chengdu 610083, China \\ ${ }^{4}$ Department of Function, Sichuan Petroleum General Hospital, Chengdu 610212, China
}

Correspondence should be addressed to Yongjian Yang; yyj10001@126.com and Haifeng Pei; web2010@foxmail.com

Received 12 March 2019; Accepted 23 July 2019; Published 17 October 2019

Guest Editor: Tatjana Bačun

Copyright (C) 2019 Jin Du et al. This is an open access article distributed under the Creative Commons Attribution License, which permits unrestricted use, distribution, and reproduction in any medium, provided the original work is properly cited.

Background. Hyper-free fatty acidemia (HFFA) impairs cardiac capillaries, as well as type 2 diabetes mellitus (T2DM). Perilipin 5 (Plin5) maintains metabolic balance of free fatty acids (FFAs) in high oxidative tissues via the states of nonphosphorylation and phosphorylation. However, when facing to T2DM-HFFA, Plin5's role in cardiac microvascular endothelial cells (CMECs) is not defined. Methods. In mice of WT or Plin ${ }^{-1-}$, T2DM models were rendered by high-fat diet combined with intraperitoneal injection of streptozocin. CMECs isolated from left ventricles were incubated with high glucose (HG) and high FFAs (HFFAs). Plin5 phosphorylation was stimulated by isoproterenol. Plin5 expression was knocked down by small interfering RNA (siRNA). We determined cardiac function by small animal ultrasound, apoptotic rate by flow cytometry, microvessel quantity by immunohistochemistry, microvascular integrity by scanning electron microscopy, intracellular FFAs by spectrophotometry, lipid droplets (LDs) by Nile red staining, mRNAs by quantitative real-time polymerase chain reaction, proteins by western blots, nitric oxide (NO) and reactive oxygen species (ROS) by fluorescent dye staining and enzyme-linked immunosorbent assay kits. Results. In CMECs, HFFAs aggravated cell injury induced by HG and activated Plin5 expression. In mice with T2DM-HFFA, Plin5 deficiency reduced number of cardiac capillaries, worsened structural incompleteness, and enhanced diastolic dysfunction. Moreover, in CMECs treated with HG-HFFAs, both ablation and phosphorylation of Plin5 reduced LDs content, increased intracellular FFAs, stimulated mitochondrial $\beta$-oxidation, added ROS generation, and reduced the expression and activity of endothelial nitric oxide synthase (eNOS), eventually leading to increased apoptotic rate and decreased NO content, all of which were reversed by $\mathrm{N}$-acetyl-L-cysteine. Conclusion. Plin 5 preserves lipid balance and cell survival in diabetic CMECs by regulating FFAs metabolism bidirectionally via the states of nonphosphorylation and phosphorylation.

\section{Introduction}

The main features of type 2 diabetes mellitus (T2DM) are hyperglycemia and metabolic disturbance of lipids and proteins $[1,2]$. More than $67.1 \%$ of T2DM patients have abnormal lipid metabolism [3], such as hyper-free fatty acidemia (HFFA) and hypercholesterolemia. Free fatty acids (FFAs), known as nonesterified fatty acids (NEFAs), are closely related to metabolic syndrome in T2DM patients [4] and serve as an important risk factor in cardiovascular diseases [5]. Moreover, within the body, the metabolic processes of glucose and lipid can interact with each other. Lee et al. even have found that plasma FFAs begin to rise progressively as earlier as two weeks prior to hyperglycemia [6]. It is clear that hyperglycemia aggrandizes the vulnerability of endothelial cells to exogenous stimulus, which can be aggravated by hyperlipidemia. Thus, we cannot ignore the interaction of glucose and lipid in research of diabetic complications. It is well known that microvascular lesions widely occurred in T2DM, resulting in abnormal microcirculation [7, 8]. Microvascular endothelial cells form the basic structure of microvessels, and the pathological changes in cardiac microvascular endothelial 
cells (CMECs) occur before the myocardium in the diabetic heart [9]. Recently, coronary microvascular diseases (CMD) have attracted wide attention, such as cardiac X syndrome, coronary slow flow phenomenon, and no-reflow phenomenon. By using coronary angiography, it is believed that microvascular endothelial dysfunction is an important cause of CMD [10]. Therefore, it is an urgent task to explore the molecular mechanism of CMECs injury, which may help develop new target for CMD.

Lipid droplets (LDs) serve as essential organelles for intracellular lipid storage [11]. Perilipin 5 (Plin5) is one of the important members of lipid droplet-associated proteins on the surface of LDs and is highly expressed in high fatty acid oxidation tissues, such as heart, skeletal muscle, liver, and brown fat [12]. Plin5 plays an important role in cellular lipid metabolism by promoting or inhibiting the hydrolysis of neutral fat in LDs. It not only ensures the energy supply of cells but also prevents the lipid toxicity damage caused by fatty acid overload [13]. A large amount of literatures reveal that Plin5 knockout reduces the formation of LDs, enhances the oxidation of FFAs, and causes insulin resistance [14-16]. In vascular endothelial cells, LDs can be quickly formed and decomposed [17]. Plin5 deletion in large arteries of mice can accelerate the progression of atherosclerosis [18]. On the contrary, it is reported that Plin5 overexpression with high cardiac specificity results in myocardial steatosis and mitochondrial dysfunction in mice $[19,20]$. However, the concrete role of Plin5 in CMECs has not been well defined yet. More interestingly, the phosphorylation mode of Plin5 (p-Plin5) can promote the hydrolysis of triglyceride (TG) in LDs and enhance the release of FFAs into the cytoplasm [21]. Many studies have shown that protein kinase A (PKA) activation can stimulate the phosphorylation of Plin5 [22, 23], in which serine 155 may be the important site [22, 24]. Moreover, p-Plin5 in the nucleus can also inhibit SIRT1 activity, promote the transcription of peroxisome proliferator-activated receptor gamma coactivator $1-\alpha$ (PGC- $1 \alpha$ ), and eventually enhance the synthesis and oxidation function of mitochondria [24]. Furthermore, it is reported that increasing the PKA-mediated phosphorylation of Plin5 by atorvastatin can reduce cellular lipid accumulation in hepatocytes [25]. However, the specific action of p-Plin5 in diabetic CMECs remains unclear.

Oxidative stress is caused by excessive production of reactive oxygen species (ROS) and reactive nitrogen (RNS) and/or the reduction of cellular antioxidant capacity [26, 27]. Mitochondria is the most important source of ROS in cells [28], and the $\beta$-oxidation of FFAs can produce a large number of ROS $[29,30]$. Many studies show that Plin5 is closely related to oxidative stress, and Plin5 knockout can lead to enhanced mitochondrial $\beta$-oxidation in myocardium and hepatocytes in mice $[16,31]$. However, whether oxidative stress mediates the biological function of Plin5/p-Plin5 in diabetic CMECs deserves a thorough study.

The aims of this study are to determine (1) whether Plin5 is disturbed in diabetic CMECs, (2) whether the deficiency and phosphorylation of Plin 5 affect the survival of CMECs under the condition of T2DM-HFFA, and, if so, (3) to iden- tify whether oxidative stress participates in those biological effects of Plin5/p-Plin5 in CMECs.

\section{Material and Methods}

2.1. Preparation of Mice. Normal mice of wild type (WT; C57BL6/J, male) were obtained from Vital River (Beijing, China) and Plin $5^{-/-}$mice from Graduate School of Life Science (Hyogo, Japan) for this experiment [14]. The reverse transcription-polymerase chain reaction (RT-PCR) was used to detect the genotypes of mice. Mice were given free access to water and feed and raised under standard conditions at $22-26^{\circ} \mathrm{C}$ with 12 -hour light/12-hour dark cycle. These two genotypes mice were both randomly divided into the control group and type 2 diabetes mellitus with hyper-free fatty acidemia (T2DM-HFFA) group. To prepare animal models of T2DM-HFFA, 6-week-old mice were given high-fat diet (60\% from fat, $20 \%$ from carbohydrates, and $20 \%$ from protein, Research Diets, USA) for 8 weeks and then were fasted for 16 hours and subsequently given intraperitoneal injection of streptozocin (STZ; $30 \mathrm{mg} / \mathrm{kg}$, Sigma, USA). Later, these mice were fed continuously with high-fat diet for 16 weeks [32]. The fasting blood glucose levels $\geq 11.1 \mathrm{mmol} / \mathrm{L}$ and with symptoms of polyuria and polydipsia were confirmed to be successful in modeling [33]. Meanwhile, serum FFAs level $\geq 3.0 \mathrm{mmol} / \mathrm{L}$ was defined HFFA [34]. 24 weeks later, mice with hyperglycemia and HFFA were used for the experiments. All experiments were conducted in adherence with National Institutes of Health Guidelines on the Use of Laboratory Animals and were approved by the Institutional Animal Care and Use Committee in the General Hospital of Western Theater Command.

2.2. Biochemical Indicator. The mice were given 12 hours of fasting treatment before experiments. Electronic balance was used to detect body weight of mice. Serum levels of glucose, insulin (INS), free fatty acids (FFAs), triglyceride (TG), and cholesterol (CHOL) were determined by automatic biochemical analyzer (Model 7020, Hitachi, Japan).

2.3. Scanning Electron Microscope. Mice were anesthetized with $4 \%$ chloral hydrate, and the abdominal aorta was intubated. Heparin saline and acetone butanone mixture was perfused in turn. Then, acrylonitrile butadiene styrene (ABS; $10 \%$ ) solution was perfused into for $15 \mathrm{~mL}$ when perfusion pressure was maintained at about $200 \mathrm{mmHg}$. After the perfusion was completed, the hearts of mice were placed at room temperature for 24 hours and then corroded by concentrated hydrochloric acid for 1 week. Microvascular casts were obtained by rinsing with water and dried by freezedrying. After spraying with gold, the microvasculatures were observed by the scanning electron microscope (Model S$3400 \mathrm{~N}$, Hitachi, Japan) at $15 \mathrm{kV}$.

2.4. Immunohistochemical Staining. After fixed with formaldehyde, the heart was given treatment of paraffin embedding and finally made into slices with a thickness of $5 \mu \mathrm{m}$. Sections were incubated with hydrogen peroxide $\left(\mathrm{H}_{2} \mathrm{O}_{2}\right.$; $3 \%, 25$ minutes) to eliminate the endogenous peroxidase. Then, sections were covered with bovine serum albumin 
(BSA; 3\%, 30 minutes) at room temperature. After the rabbit anti-mouse CD31 primary antibody $(1: 200$, CST, USA) was added, sections were kept in the wet box $\left(4^{\circ} \mathrm{C}\right.$, overnight). After washed with phosphate-buffered saline (PBS), goat anti-rabbit secondary antibody ( $1: 1000$, CST, USA) was added and incubated at $37^{\circ} \mathrm{C}$ for 2 hours. The sections were observed under an optical microscope (DM3000, Leica, Germany). The microvessels were the standard of blood vessel with diameter less than $100 \mu \mathrm{m}$. Each slice was randomly observed for 5 visual fields, and the mean numbers of microvessels were calculated with Image-Pro Plus.

2.5. Cardiac Function. The mice were anesthetized with $4 \%$ chloral hydrate. VEVO 2100 high-resolution in vivo imaging system (Visual Sonics, Canada) was used to measure the left ventricular early mitral diastolic wave/late mitral diastolic wave (E/A) ratio and the percentage of left ventricular ejection fraction (LVEF). The whole detection process was carried out by an ultrasonic technician who was blinded to the experimental groups, and the detection parameters remained unchanged.

2.6. Preparation of CMECs. CMECs were isolated from the hearts as previously described $[35,36]$. Briefly, the hearts of experimental mice were washed with precooled PBS at $4^{\circ} \mathrm{C}$. After the aorta and atrial tissues were dislodged, the epicardium and endocardium were removed from ventricular tissues which were then cut into slices of $1 \mathrm{~mm}^{3}$ size pieces and incubated in collagenase II (0.2\%, Sigma, USA) for 30 minutes. After that, $100 \mu \mathrm{m}$ strainer mesh was used to remove the undigested tissues. Finally, cells were cultured in the Dulbecco's modified Eagle's medium (DMEM; HyClone, USA) supplemented with fetal bovine serum (FBS; 15\%, Gibco, USA). The cells for transplantation were purified using differential time adherent method. Once cell confluence reaches $80 \%$, cells were digested with trypsin $\left(0.25 \%, 37^{\circ} \mathrm{C}, 20\right.$ minutes; Sigma, USA). The third to fifth generation cells were harvested for the further experiments. CMECs were positively identified by staining of von Willebrand factor (vWF) and CD31. Media were replaced to different conditions 24 hours after cell inoculation: normal glucose medium (normal, $5.5 \mathrm{mmol} / \mathrm{L}$ ), high glucose medium (HG, $25 \mathrm{mmol} / \mathrm{L}$ ), high free fatty acids medium (HFFAs; $1 \mathrm{mmol} / \mathrm{L}$, Sigma, USA), and high glucose plus high free fatty acids medium (HG-HFFAs). HFFAs were a mixture of palmitate and oleate, $1: 2(w / w)$ [37]. Then, the cells were incubated for another 24 hours. Reagents and drugs used in cell experiments in vitro were as follows: $\mathrm{N}$-acetyl-L-cysteine (NAC; $10 \mathrm{nmol} / \mathrm{L}, 8$ hours; Sigma, USA) and isoprenaline (ISO; $10 \mu \mathrm{mol} / \mathrm{L}, 8$ hours; Sigma, USA).

2.7. Cellular Immunofluorescence. CMECs were fixed in paraformaldehyde at room temperature for 30 minutes. Triton $(0.1 \%, 5$ minutes) was used to broken film. Then, cells were covered with 3\% BSA for 30 minutes. Rabbit anti-mouse Plin5 primary antibody (1:500, Novus, USA) was added and kept overnight at $4^{\circ} \mathrm{C}$. The donkey anti-rabbit fluorescent secondary antibody $(1: 1000$, Abcam, USA) was added the next day, and the cells were observed under laser confocal microscopy (LSCM; FV 1000, Olympus, Japan) after cleaning with PBS for 3 times.

2.8. Cell Apoptosis. Cell apoptosis was measured by Annexin V-FITC/PI assay kit (BD, USA). After digestion, CMECs were washed 2 times with cooled PBS. Then, cells were suspended with $1 \times$ binding buffer and added with Annexin V-FITC and PI $2.5 \mu \mathrm{L}$ each. CMECs were incubated under light avoidance conditions at room temperature for 15 minutes. Apoptosis was detected by flow cytometry (Partec, Germany), and data were analyzed by FlowJo software.

2.9. Nitric Oxide (NO). When the intervention was completed, CMECs were incubated with DAF-2DA, a kind of fluorescent probe for the detection of $\mathrm{NO}(5 \mu \mathrm{mol} / \mathrm{L}$, 20 minutes; Abcam, England). After washed by PBS for 3 times to remove the unbound DAF-2DA, CMECs were observed under laser confocal microscopy (LSCM; FV 1000, Olympus, Japan) at $488 \mathrm{~nm}$ emission wavelengths. In addition, the level of $\mathrm{NO}$ in CMECs was also detected by enzyme-linked immunosorbent assay (ELISA) kit (Elixir, Canada) following the instruction [38].

2.10. Intracellular ROS. According to the $1: 1000$ ratio, dihydroethidium (DHE; Beyotime, China) was diluted with Hank's balanced salt solution (HBSS; Invitrogen, USA) to make the final concentration $(5 \mu \mathrm{mol} / \mathrm{L})$. CMECs were incubated with $\mathrm{DHE}$ in the incubator of $37^{\circ} \mathrm{C}$ for 45 minutes. After washed with HBSS, cells were observed under fluorescence microscope (Olympus, Japan). Besides, the content of intracellular ROS was also detected by ELISA kit (Elixir, Canada) following the instruction [39].

2.11. Gene Expression. Total RNA was extracted from cells using TRIzol reagent (Invitrogen, USA), quantitated using a NanoDrop 2000 spectrophotometer. Then, the RNA was reverse-transcribed using a kit (TaKaRa, Japan). Quantitative real-time polymerase chain reaction (qRT-PCR) was performed in a CFX96 real-time detection system (Bio-Rad, USA) using SYBR Green PCR kit (TaKaRa, Japan). Relative mRNA levels were quantified using the comparative $\Delta \Delta \mathrm{CT}$ method with GAPDH as reference gene. The following primer sequences were used for qRT-PCR: GAPDH forward, 5'-AGGTCGGTGTGAACGGATTTG-3' , reverse, 5' -TGTA GACCATGTAGTTGAGGTCA-3'; Plin5 forward, 5'-GA AGTGGGCACAGTGGAGG-3', reverse, 5' -AAAGAGTGT TCATAGGCGAGAT- $3^{\prime}$; and carnitine palmitoyl transferase 1 (CPT-1) forward, $5^{\prime}$-CACTGCAGCTCGCACATTA C-3', reverse, $5^{\prime}$-CCAGCACAAAGTTGCAGGAC-3'.

2.12. Western Blot. CMECs were washed with PBS for 3 times; total protein and nuclear protein were extracted by a kit (Sigma, USA). The concentration of protein was determined with the Bio-Rad protein assay kit (Bio-Rad, USA). Equivalent amounts of total protein $(25 \mu \mathrm{g})$ were subjected to SDS-PAGE and transferred to PVDF membranes. The membranes were incubated with specific primary antibodies against Plin5 (1:2000, Novus, USA), GAPDH $(1: 3000$, Novus, USA), Histone H3 ( $1: 2000$, CST, USA), and eNOS 
( $1: 2000$, Novus, USA) for overnight. After incubation with goat anti-rabbit IgG secondary antibody $(1: 2000,1$ hour; Thermo Fisher, USA) at room temperature, proteins were detected with enhanced chemiluminescence and quantified using Image-Pro Plus software [40].

2.13. Nile Red Staining. The cells were rinsed 2 times with PBS and fixed by incubating with $2 \mathrm{~mL} 4 \%(w / v)$ paraformaldehyde at room temperature for 30 minutes. After that, the cells were washed 3 times with PBS. Nile red stock solution $(1 \mathrm{mg} / \mathrm{mL}, 1 \mu \mathrm{L})$ was added to $10 \mathrm{~mL}$ of PBS. Then, the cells were covered with $1 \mathrm{~mL}$ Nile red staining solution and incubated for 10 minutes, all of which were protected from ambient light. The cells were washed with PBS 3 times and observed under laser confocal microscopy. The fluorescence intensity was analyzed by ImageJ software.

2.14. Intracellular FFAs. CMECs were homogenized in $200 \mu \mathrm{L}$ of $1 \%(w / v)$ Triton X-100 in chloroform solution. The samples were centrifuged at $13,000 \mathrm{~g}$ for 10 minutes to remove insoluble material. We collected the organic phases (lower phase) and removed chloroform by air dry at $50^{\circ} \mathrm{C}$. The dried lipids were dissolved in $200 \mu \mathrm{L}$ fatty acid assay buffer by vortexing extensively for 5 minutes. Then, the concentration of FFAs was determined by a Free Fatty Acid Quantitation Kit (Sigma, USA), according to the instruction.

2.15. siRNA Transfection. One day before transfection, the cells were inoculated into 6-well plates and cultured in antibiotic-free medium. Moderate transfection reagent was added Opti-MEM medium and incubated at room temperature for 5 minutes. Small interfering RNA (siRNA), including the control siRNA and Plin5 siRNA (Invitrogen, USA), was added into Opti-MEM medium and blended. Then, the diluted siRNA was gently mixed with the incubated transfection reagent and incubated for 30 minutes at room temperature. Next, we removed the previous medium from the 6-well plates, and added $1.5 \mathrm{ml}$ new antibiotic-free medium and $500 \mu \mathrm{l}$ siRNA-transfection reagent in it. The cells were placed in the incubator for 48 hours and then intervened as planned.

2.16. Statistical Analyses. Data were shown as means \pm standard errors of the means (SEM). The independent sample $t$-test was used in the comparison between the 2 groups. The single-factor ANOVA was used in comparison among multiple groups, and pairwise comparison in multiple groups was conducted with LSD $t$-test. $P \leq 0.05$ was considered significant. All statistical tests were performed using GraphPad Prism software version 7 .

\section{Results}

3.1. High FFAs Aggravated Cardiac Microvascular Injury Induced by HG. We established mouse models of T2DMHFFA to observe pathological changes in cardiac microvasculature and function. Compared with the control mice, serum levels of glucose, insulin, and FFAs increased significantly in diabetic mice (Table S1), revealing that we prepared the basic platform for our experiments. Echocardiography showed that E/A ratio, but not LVEF percentage, was decreased obviously in diabetic mice (Figures 1(a) and 1(b)), hinting at that T2DM gives rise to ventricular diastolic dysfunction. Moreover, in diabetic mice, the number of cardiac microvessels decreased remarkably (Figure 1(c)), and those remaining microvessels became rough and uneven (Figure 1(d)). In view of the fact that cardiac microvessel is composed of monolayer endothelial cells, CMECs were isolated from neonatal mice. More than $95 \%$ of the cells were positively identified by the endothelial cell marker, vWF and CD31 (Figure S1A). HG significantly enhanced CMECs apoptosis and reduced cellular NO content, all of which were further deteriorated by intervention of high FFAs (HFFAs) (Figures 1(e)-1(g)). We also repeated the main experiments on CMECs isolated from the control and T2DM-HFFA mice; the computational data from the T2DM-HFFA modeling largely indicated similar trends with the previous experimental results. T2DM-HFFA not only exacerbated endothelial apoptosis but also further inhibited NO generation (Figure S1B and S1C). These data suggest that disorder of FFAs metabolism is able to aggravate cardiac microvascular injury induced by HG.

\subsection{Plin5 Deletion Increased Intracellular FFAs Content in} CMECs. In order to clarify the concrete role of Plin 5 in metabolism of FFAs, immunofluorescence, qRT-PCR and western blots were introduced to judge Plin 5 changes in CMECs. As a result, HFFAs, but not HG, enhanced Plin5 expression (Figures 2(a)-2(c)). In addition, RT-PCR was used to detect genotypes in mice (Figure S2), and CMECs were isolated from the WT and Plin $5^{-1-}$ mouse hearts. Nile red staining showed that HG-HFFAs intervention increased intracellular LDs content, and Plin5 deletion significantly reduced intracellular LDs content (Figure 2(d)), whereas, compared with CMECs in WT, Plin5 deficiency had increased intracellular FFAs content under the condition of normal and HG-HFFAs (Figure 2(e)). These results reveal that Plin5 plays a critical role in the FFAs metabolism of CMECs, which may affect the survival and function of CMECs.

\subsection{Plin5 Deficiency Exacerbated CMECs Injury Induced by} HG-HFFAs. Although Plin5 is reported to protect cells from lipid toxicity via preventing excessive FFAs release from TG, its role in lipid metabolism of vascular endothelium has not been investigated. Here, we found that Plin5 knockout had no significant effects on the level of body weight, blood glucose, insulin, and blood lipid (Table S1). Echocardiographic findings suggested that Plin5 ablation had no significant effect on E/A ratio and LVEF under basic condition. However, it exacerbated ventricular diastolic dysfunction in T2DM-HFFA mice (Figures 3(a) and 3(b)). Corresponding to the ultrasound findings, imaging of scanning electron microscopy and immunohistochemistry showed that Plin5 ablation hardly affected the number and integrity of cardiac microvessels under basic condition, whereas Plin5 deletion resulted in less number of cardiac microvessels and worse endothelial junctions between CMECs under T2DM-HFFA condition (Figures 3(c) and 3(d)). Moreover, cell apoptosis and NO production in WT were close to those in Plin $5^{-1-}$ under normal condition. But, Plin5 knockout in CMECs 

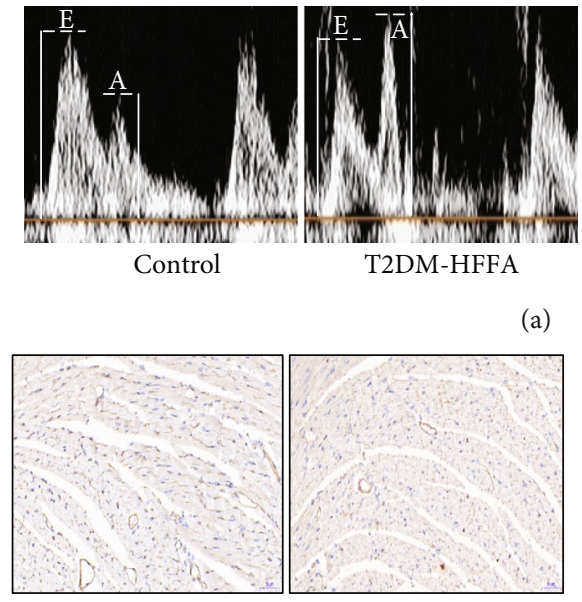

Control

T2DM-HFFA (a)
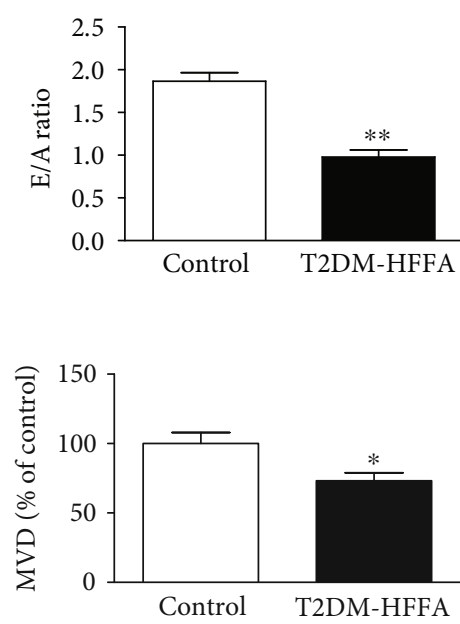

(c)

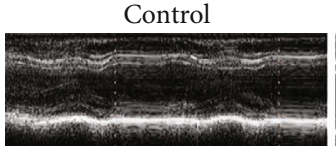

T2DM-HFFA
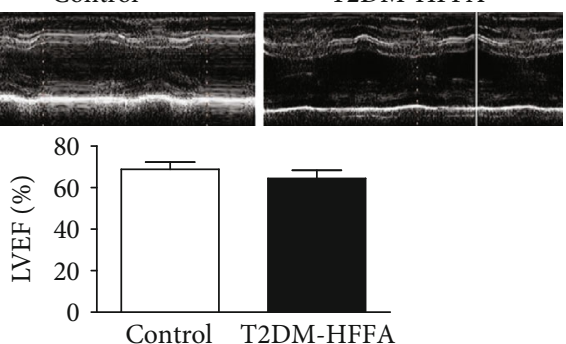

(b)

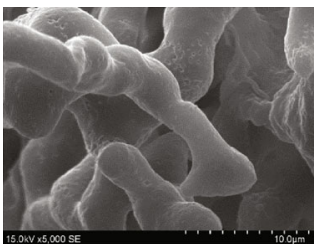

Control

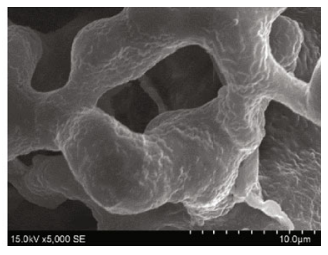

T2DM-HFFA

(d)
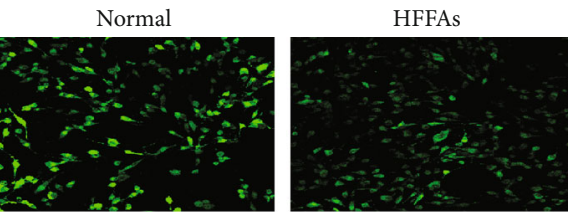

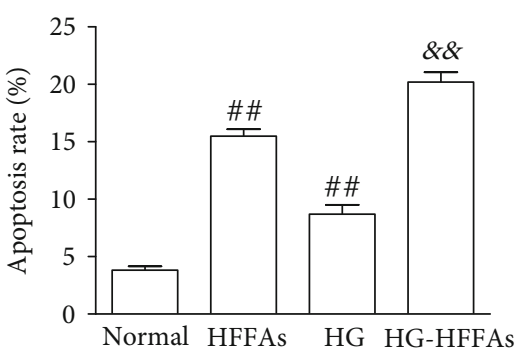

(e)

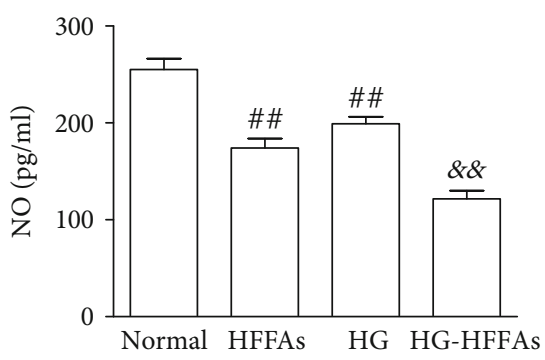

(f)
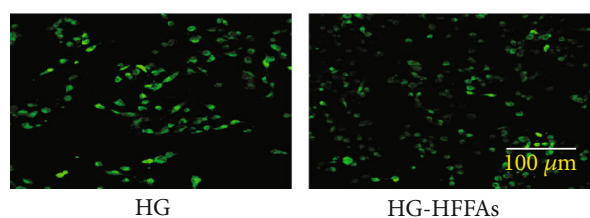

(g)

Figure 1: The influence of HG-HFFAs on CMECs. (a) The representative mitral flow patterns detected by cardiac ultrasound (left); the quantification of E/A ratio (right). (b) The percentage of LVEF detected by cardiac ultrasound. (c) The number of cardiac microvessels counted by CD31 immunohistochemical staining (left); the quantification of MVD (right). (d) Cardiac microvascular integrity detected by scanning electron microscope. (e) The apoptosis rate measured by Annexin V-FITC/PI assay kit. (f) NO generation in CMECs measured by ELISA kit. (g) NO production in CMECs detected by staining with DAF-2DA. T2DM-HFFA: type 2 diabetes mellitus with hyper-free fatty acidemia; HG: high glucose; HFFAs: high free fatty acids; HG-HFFAs: high glucose and high free fatty acids; E/A: early mitral diastolic wave/late mitral diastolic wave; LVEF: left ventricular ejection fraction; MVD: microvessel density; ELISA: enzyme-linked immunosorbent assay. Data are expressed as mean \pm SEM, $n=6$-8/group. ${ }^{*} P<0.05,{ }^{* *} P<0.01$ vs. control; ${ }^{\# \#} P<0.01$ vs. normal; ${ }^{\& \&} P<0.01$ vs. HG.

exacerbated cell apoptosis and NO reduction induced by HG-HFFAs (Figures 3(e)-3(g)). siRNA was used to knock down the expression of Plin5 in WT; results showed that Plin5 knockdown had no significant effect on the apoptotic rate and NO production in CMECs under normal conditions, and yet it aggravated damage to CMECs under HG-HFFAs conditions (Figure S3A and $\mathrm{S} 3 \mathrm{~B})$. In order to explore the mechanism of decreasing NO production, the activity and protein content of endothelial nitric oxide synthase (eNOS) were detected. Under normal conditions, Plin5 knockout had little effect on the activity and protein content of eNOS in CMECs. Nevertheless, under HG-HFFAs conditions, it did reduce the activity and content of eNOS in CMECs (Figure S4A and S4B). Thus, Plin5 serves as an essential molecule in the survival and function of CMECs when facing to the insult of HG-HFFAs.
3.4. Plin5 Phosphorylation Worsened CMECs Injury Induced by HG-HFFAs. Plin5 phosphorylation also plays an important role in FFAs metabolism. Here, we introduced ISO to activate the phosphorylation of Plin5. With the western blot technology, we found that ISO intervention added endonuclear Plin5 expression in CMECs under normal and HGHFFAs conditions (Figure 4(a)), which indirectly indicated that cellular Plin5 phosphorylation was enhanced. Meanwhile, after administration of ISO in CMECs, FFAs level significantly increased and LDs content obviously decreased, under normal and HG-HFFAs conditions (Figures 4(b) and 4(c)). Moreover, ISO intervention also deteriorated cell apoptosis and depressed NO generation in CMECs under HG-HFFAs condition. But it is important to note that ISO intervention had no obvious effect on apoptotic rate and NO production under normal condition (Figures 4(d)$4(f))$. Further detection found that ISO intervention had 

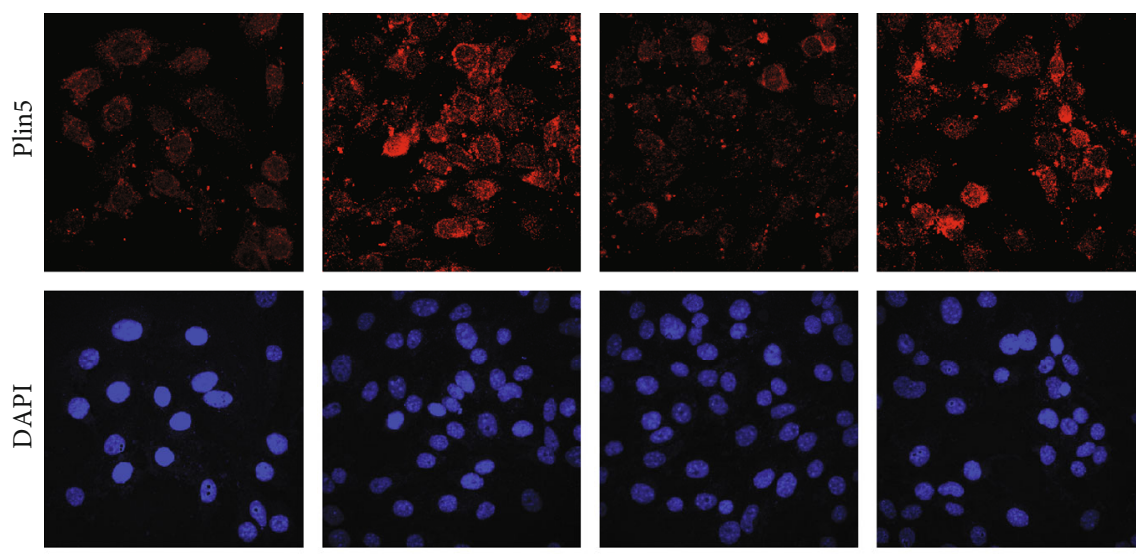

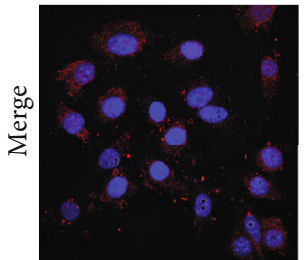

Normal

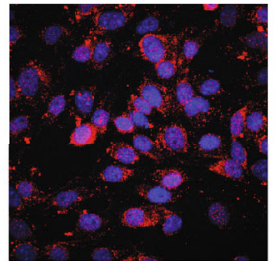

HFFAs

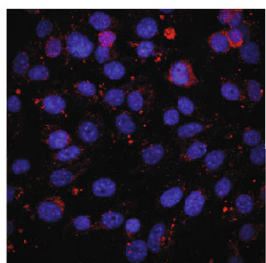

HG

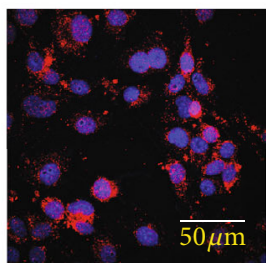

HG-HFFAs

(a)
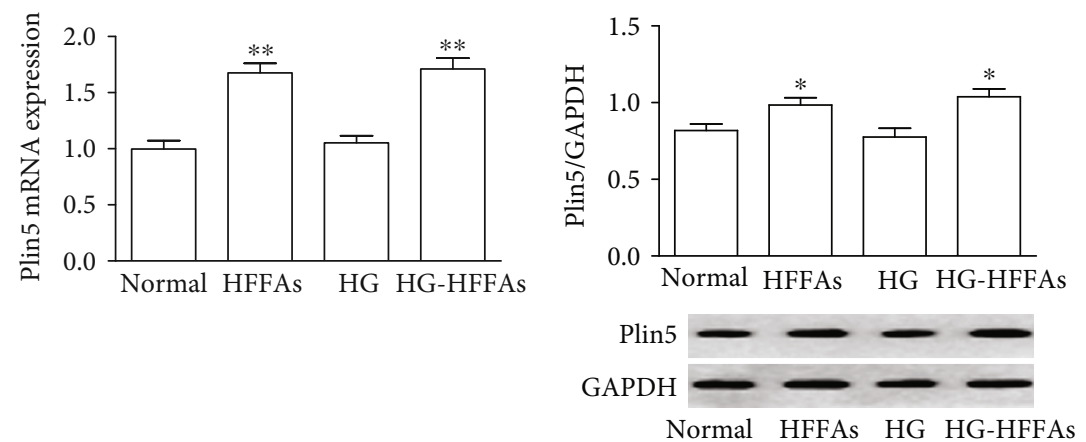

(c)
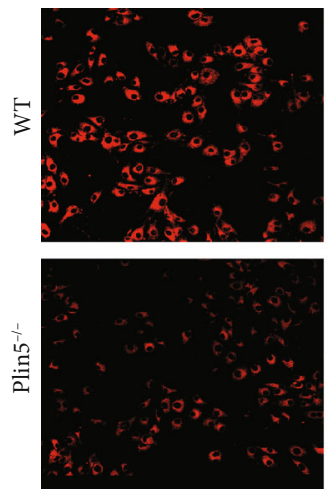

Normal
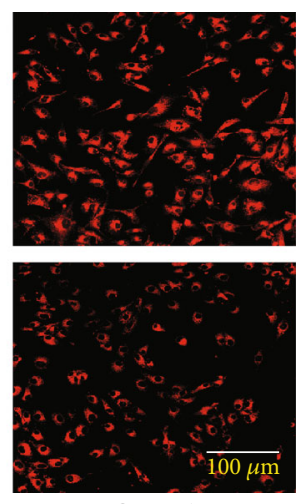

HG-HFFAs

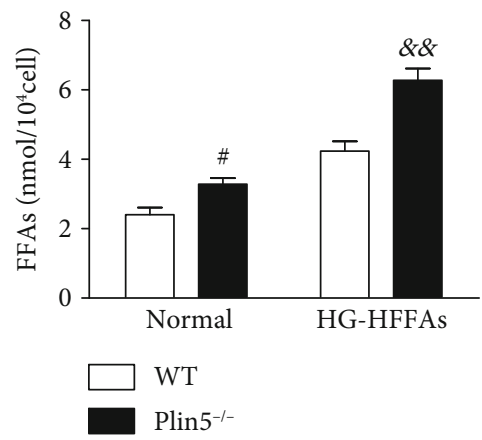

(d)

FIgure 2: The effects of HG or HFFAs on Plin5 expression in CMECs. (a) Plin5 expression detected by immunofluorescence staining. (b) The mRNA level of Plin 5 in CMECs measured by qRT-PCR. (c) The protein level of Plin5 in CMECs determined by western blot. (d) The content of LDs in CMECs detected by Nile red staining. (e) The intracellular level of FFAs in CMECs measured by Free Fatty Acid Quantitation Kit. qRT-PCR: quantitative real-time polymerase chain reaction; HG: high glucose; HFFAs: high free fatty acids; HG-HFFAs: high glucose and high free fatty acids; DAPI: $4^{\prime}, 6$-diamidino-2-phenylindole. Data are expressed as mean \pm SEM, $n=6-8$ /group. ${ }^{*} P<0.05$ vs. normal; ${ }^{* *} P<0.01$ vs. normal; ${ }^{*} P<0.05$ vs. WT in normal; ${ }^{\& \&} P<0.01$ vs. WT in HG-HFFAs. 

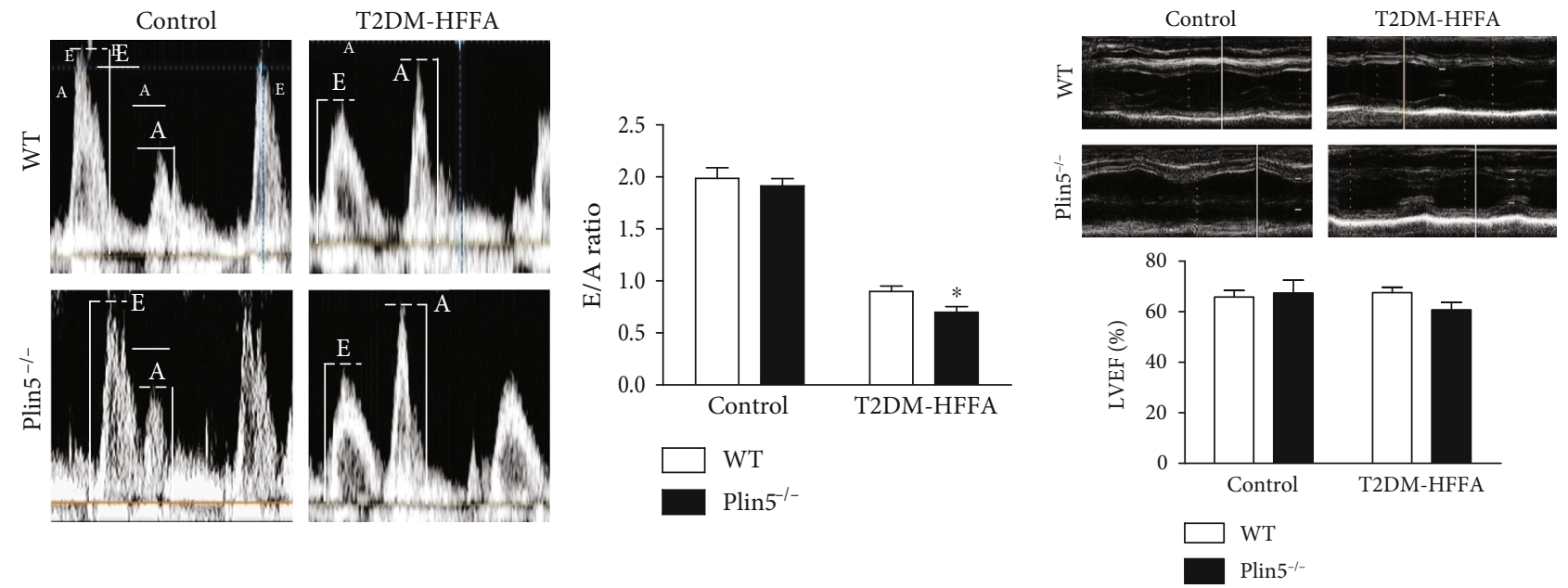

(a)

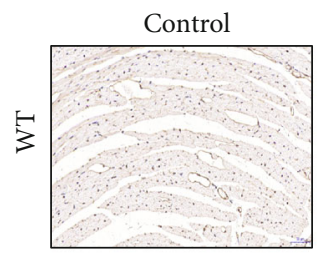

T2DM-HFFA
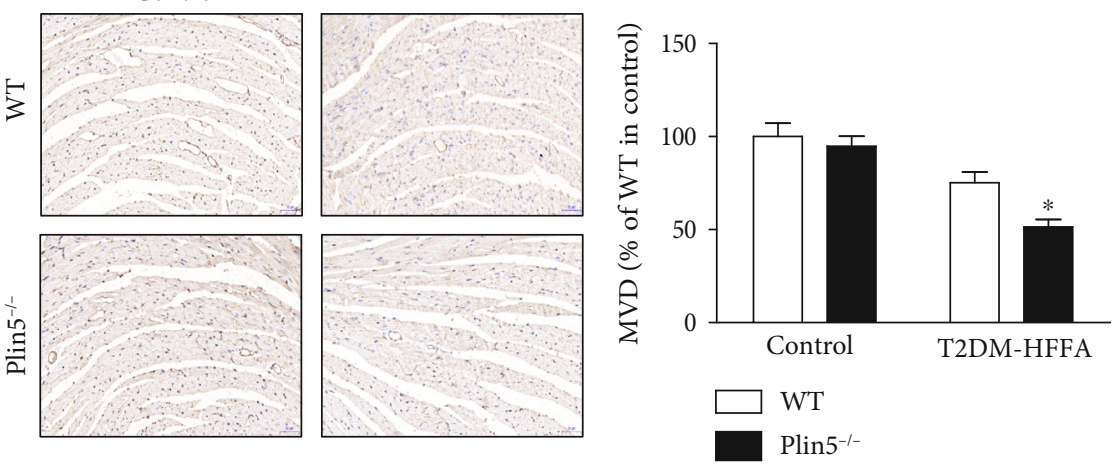

(c)

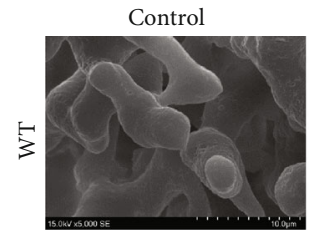

T2DM-HFFA
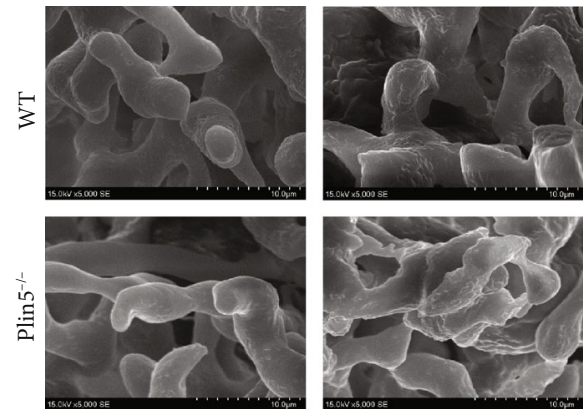

(d)

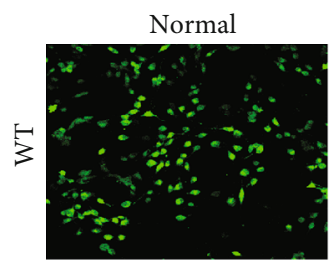

HG-HFFAs

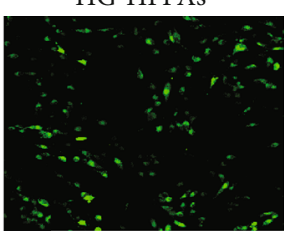

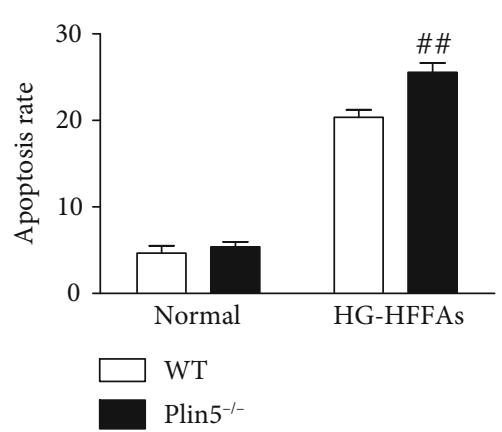

(e)

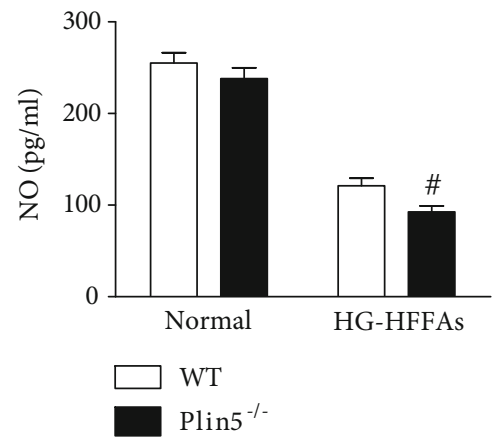

(f)

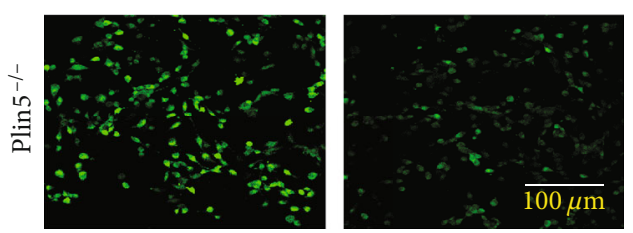

(g)

Figure 3: The influence of Plin5 deficiency on CMEC injury induced by HG-HFFAs. (a) The representative mitral flow patterns detected by cardiac ultrasound (left); the quantification of E/A ratio (right). (b) The percentage of LVEF detected by cardiac ultrasound. (c) The number of cardiac microvessels counted by CD31 immunohistochemical staining (left); the quantification of MVD (right). (d) Cardiac microvascular integrity detected by scanning electron microscope. (e) The apoptosis rate measured by Annexin V-FITC/PI assay kit. (f) NO generation in CMECs measured by ELISA kit. (g) NO production in CMECs detected by staining with DAF-2DA. WT: wild type; T2DM-HFFA: type 2 diabetes mellitus with hyper-free fatty acidemia; HG: high glucose; HFFAs: high free fatty acids; HG-HFFAs: high glucose and high free fatty acids; E/A: early mitral diastolic wave/late mitral diastolic wave; LVEF: left ventricular ejection fraction; MVD: microvessel density; ELISA: enzyme-linked immunosorbent assay. Data are expressed as mean \pm SEM, $n=6$-8/group. ${ }^{*} P<0.05$ vs. WT in T2DM-HFFA; ${ }^{\#} P<0.05,{ }^{\# \#} P<0.01$ vs. WT in HG-HFFAs. 

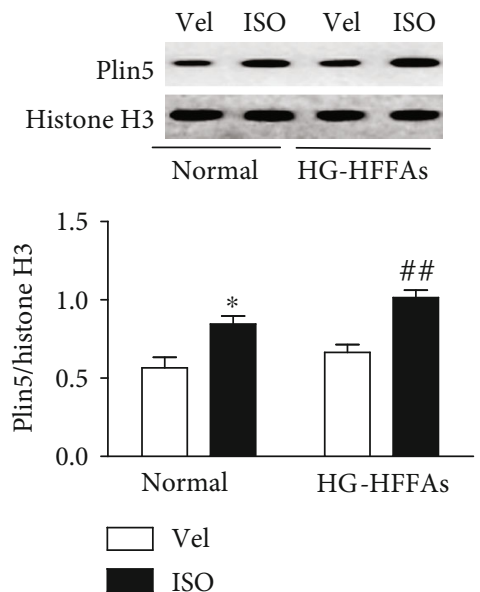

(a)

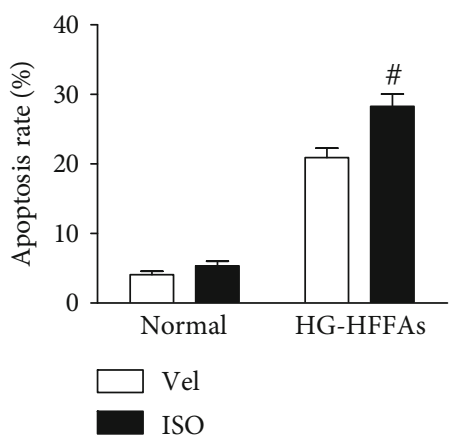

(d)

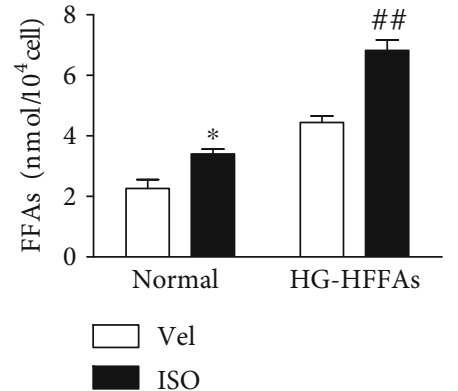

(b)

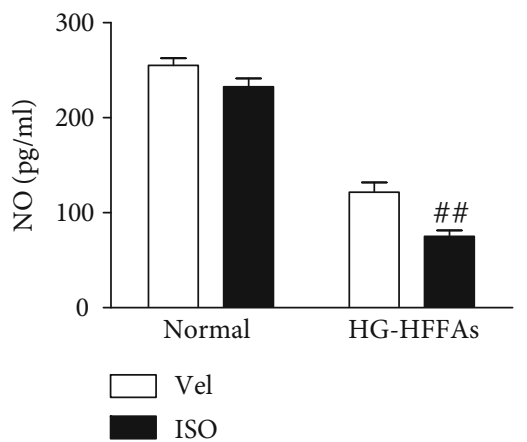

(e)
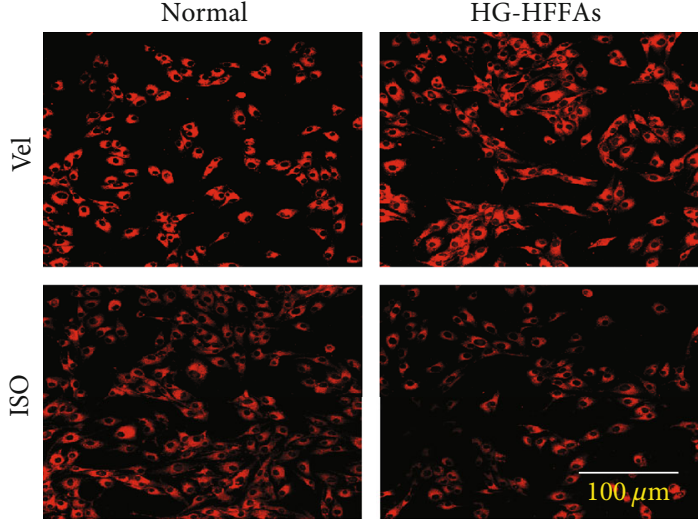

(c)
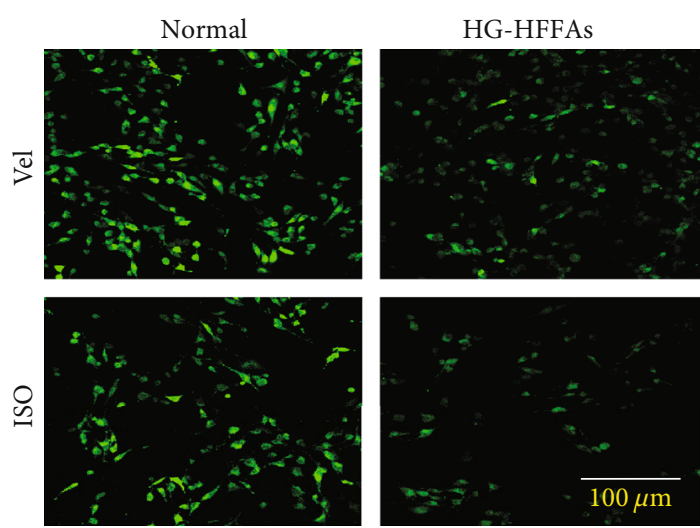

(f)

Figure 4: The influence of p-Plin5 on CMECs injury induced by HG-HFFAs. (a) The protein level of Plin5 detected by western blot. (b) The intracellular FFAs content measured by Free Fatty Acid Quantitation Kit. (c) The content of LDs in CMECs determined by Nile red staining. (d) The apoptosis rate. (e) The production of NO measured by ELISA kit. (f) NO generation detected by staining with DAF-2DA in CMECs. Vel: vehicle; ISO: isoproterenol; HG-HFFAs: high glucose and high free fatty acids; ELISA: enzyme-linked immunosorbent assay. Data are expressed as mean \pm SEM, $n=6$-8/group. ${ }^{*} P<0.05$ vs. Vel in normal; ${ }^{\#} P<0.05,{ }^{\# \#} P<0.01$ vs. Vel in HG-HFFAs.

little effect on the activity and protein content of eNOS under normal condition; however, it further reduced the activity and content of eNOS in CMECs under HG-HFFAs conditions (Figure S5A and S5B). In a word, the phosphorylation of Plin5 induced by sympathetic excitement will result in damage to CMECs by enhancing FFAs catabolism.

\subsection{ROS Overload Induced by Disruption in Plin5 Balance} Contributed to CMECs Injury under the Condition of HGHFFAs. It is well known to all that oxidative stress plays a key role in the development of diabetic cardiovascular complications. ROS, as the main activating factor of oxidative stress, is mainly produced by mitochondria. In this study, we explored whether intracellular ROS was affected by the deletion or phosphorylation of Plin 5 in the presence of HG-HFFAs. The experimental results showed that both knockout and phosphorylation of Plin5 in CMECs enhanced ROS generation induced by HG-HFFAs (Figures 5(a) and 5(d), Figure S6A and S6B). Moreover, knockout and phosphorylation of Plin5 enhanced the mRNA expression of CPT-1 (Figure S6C and S6D). In contrast, NAC, a commonly used intracellular antioxidant, partly reversed CMECs injury induced by Plin 5 deletion and phosphorylation under HGHFFAs condition (Figures 5(b) and 5(c), Figures 5(e) and $5(\mathrm{f}))$. These results suggest that oxidative stress is the underlying mechanism for CMECs injury induced by Plin5 deletion and phosphorylation.

\section{Discussion}

In this study, we established animal models of T2DM-HFFA by introducing WT and Plin $5^{-/-}$mice to explore the specific 


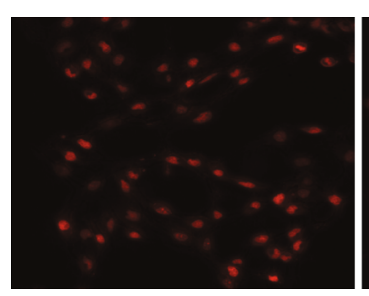
WT+Vel HG-HFFAs

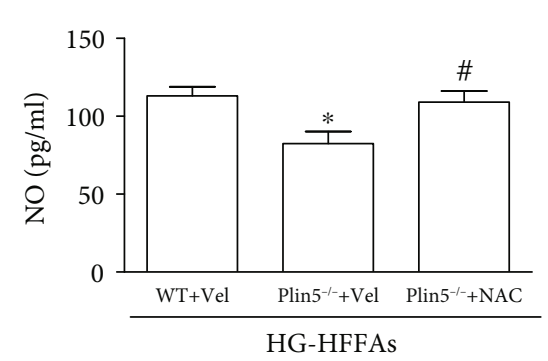

(c) (a)

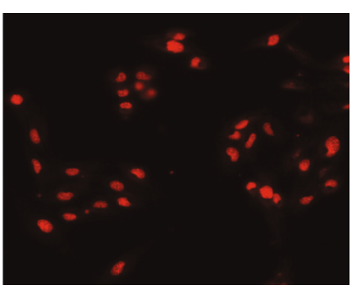

Plin5 ${ }^{-1-}+\mathrm{Vel}$

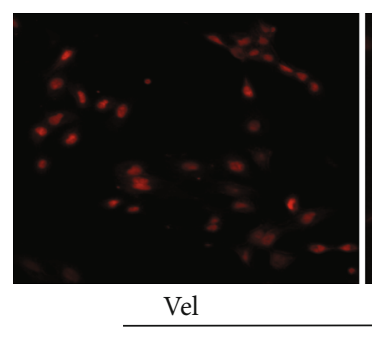

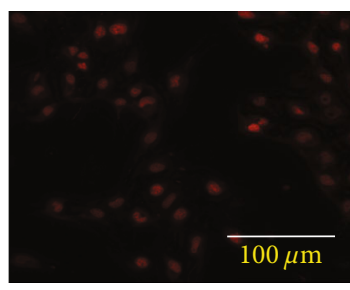
Plin5 ${ }^{-1-}+\mathrm{NAC}$

\begin{abstract}
.
\end{abstract}




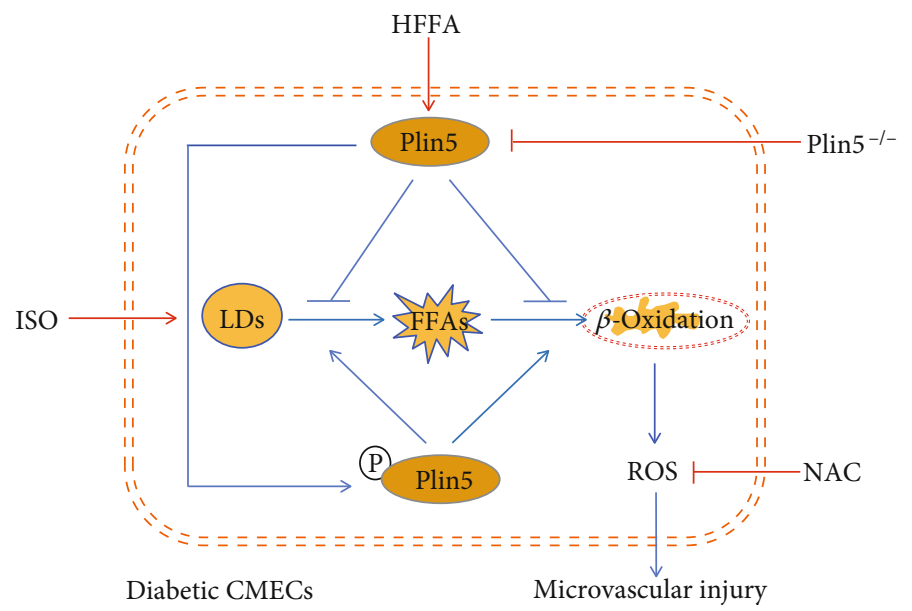

FIGURE 6: Schematic diagram depicting the bidirectional regulation of Plin5 in the metabolism of intracellular FFAs when facing to T2DMHFFA. In diabetic CMECs, HFFA, but not hyperglycemia, significantly activates Plin 5 expression, and Plin 5 is able to inhibit the hydrolysis of LDs to FFAs as well as the mitochondrial $\beta$-oxidation of FFAs, whereas Plin5 ablation promotes the hydrolysis of LDs to FFAs, enhances the mitochondrial $\beta$-oxidation of FFAs, gives rise to the excessive generation of mitochondrial ROS, and finally aggravates CMECs injury induced by T2DM-HFFA. On the other hand, the phosphorylation of Plin 5 by ISO also promotes the hydrolysis of LDs to FFAs and the mitochondrial $\beta$-oxidation of FFAs, leading to enhanced oxidative stress and deteriorated CMECs injury. ISO: isoproterenol; LDs: lipid droplets; HFFA: hyper-free fatty acidemia; T2DM-HFFA: type 2 diabetes mellitus with hyper-free fatty acidemia; NAC: N-acetyl-cysteine; ROS: reactive oxygen species; FFAs: free fatty acids.

disorders. Moreover, we found that left ventricular diastolic function was impaired in T2DM-HFFA mice, which might may be related to the disturbance of myocardial energy supply caused by microvascular injury. Furthermore, we also found that HFFAs aggravated cell apoptosis and NO reduction induced by HG in cultured CMECs. In line with our results, Pilz et al. claimed that HFFA increased the risk of ischemic heart disease and the mortality of coronary heart disease $[52,53]$, and Guo et al. defined HFFA as an independent risk factor for hypertension [54]. Some studies have reported that hyperglycemia and HFFAs induce vascular endothelial cell apoptosis and reduce the synthesis and bioavailability of NO [55-57]. Thus, it is necessary to investigate the specific mechanism of CMECs injury under the condition of HG-HFFAs and explore new targets for clinical intervention.

As mainly formed by central neutral lipids and peripheral phospholipid monolayer, LDs are key regulators for lipid metabolism both in adipose tissues and nonadipose tissues [58]. As an important member of the PAT protein family on the surface of LDs, Plin5 has aroused the enthusiasm of many researchers. Plin5 can inhibit the hydrolysis of neutral lipid in LDs through interaction with lipolytic hydrolase such as hormone-sensitive triglyceride lipase (HSL), adipose triglyceride lipase (ATGL), and comparative gene identification-58 (CGI-58), thus preventing excessive generation and oxidation of FFAs [21, 59]. In our experiments, we simulated T2DM-HFFA state in vitro by giving intervention of glucose and FFAs to cultured CMECs. As last, we found that Plin5 was expressed in CMECs derived from mice. The intervention of HFFAs, but not HG, obviously increased the expression of Plin5 at the mRNA and protein levels. Consistently, many studies proved that exogenous intervention of FFAs stimulated Plin5 expression in many kinds of cultured cells [60-62], and the increase in Plin5 may be generally attributed to the activation of peroxisome proliferator-activated receptors (PPARs) [60, 63]. Some literature even claimed that high-fat diet was able to increase Plin5 expression in the liver and skeletal muscles [16, 64, 65]. It was found that Plin 5 knockout could increase the content of FFAs in liver tissue of mice [16]. In our current studies of CMECs, we found that Plin5 ablation could reduce the content of LDs and increase the content of FFAs, suggesting that Plin5 plays an important role in the metabolism of LDs and FFAs in CMECs. Moreover, Plin5 knockout reduced the number of cardiac microvessels and aggravated the injury of microvascular integrity in T2DM-HFFA mice. In addition, Plin5 knockout aggravated cardiac diastolic dysfunction, but had little effects on cardiac systolic function in T2DM-HFFA mice. So, we speculate that the effects of Plin 5 deletion on the number and structure of cardiac microvasculature contribute to the deterioration of diastolic dysfunction. The inconformity in changes of cardiac function may be due to differences in the severity of cardiac microvascular and myocardial damage. Consistent with our data, other studies have shown that Plin5 knockout is considered to have no effect on cardiac function in the physiological state $[31,66]$, but aggravate cardiac dysfunction induced by old age or ischemic stimuli in mice [14]. Furthermore, we found that Plin5 deficiency increased apoptosis rate and decreased $\mathrm{NO}$ synthesis in CMECs under the condition of HG-HFFAs. It is well known that eNOS plays a decisive role in the production and utilization of NO in endothelial cells. Studies have confirmed that FFAs can inhibit eNOS mRNA expression and regulate eNOS activity by increasing oxidative stress and inflammatory burden in aortic endothelial cells of rats [56]. Our study also demonstrated that Plin 5 deletion reduced eNOS content in CMECs under HG-HFFAs condition, which may contributed to the reduction of NO generation. Similarly, Ibrahim 
et al. proved that in patients of obesity, diabetes and nonalcoholic fatty liver, the core of lipid toxicity was the excessive decomposition of TG to FFAs and the accumulation of FFAs [67]. Therefore, Plin5 participates in the metabolism of FFAs in CMECs and its deficiency may aggravate cardiac microvascular injury induced by T2DM-HFFA. Plin5 can be used as a new indicator to predict the occurrence and development of coronary heart disease.

Interestingly, Plin5 overexpression in the heart caused severe cardiac steatosis and left ventricular hypertrophy [19, 20]. Further study found that Plin5 overexpression did not continue to inhibit LDs decomposition in myocardium, which might be associated with p-Plin5-stimulated hydrolysis of LDs under various stressful conditions [22]. Researches in various aspects showed that PAT protein family members participated in cell lipid metabolism via two states which contained nonphosphorylation and phosphorylation. Under basic conditions, Plin5 was bound to ATGL and CGI-58, respectively, to prevent the combination of this two, thus inhibiting the hydrolysis of LDs. Plin 5 could also promote FFAs enter into LDs to synthesize neutral fat, thus preventing the excessive oxidation of FFAs [21]. On the other hand, adrenaline, secreted by sympathetic excitability in the case of cold, tension, fear, and so on, is able to activate PKA and increase the phosphorylation of Plin5, which may prompt the incidence of cardiovascular disease, especially in T2DM patients. When adrenergic receptors were activated, Plin5 was phosphorylated by PKA activation and promoted the hydrolysis of LDs. Catecholamine stimulation could increase the lipolysis rate by 50 times in adipose tissues [68]. In addition, $\mathrm{p}$-Plin 5 also participated in the transfer of FFAs to mitochondrial for $\beta$-oxidation $[13,22]$. The released FFAs from LDs could also increase the transcriptional of PPARs which stimulate gene transcription related to the synthesis and oxidation of mitochondria $[21,24]$. At present, there is no specific antibody to detect the phosphorylation of Plin5, but it has been reported that only p-Plin 5 can enter into the nucleus to play a corresponding role [24]. Our studies revealed that, in WT group, ISO stimulation increased Plin5 expression in the nucleus. More importantly, ISO enhanced the hydrolysis of LDs, raised the intracellular level of FFAs, reduced intracellular eNOS level, and exacerbated CMECs injury induced by HG-HFFAs. It should be noted that although ISO stimulation increased intracellular FFAs levels in basic condition, it did not cause damage to CMECs which may be related to cellular self-regulation. These results indicated that ISO intervention was able to increase the phosphorylation of Plin5 in CMECs and aggravate CMECs injury induced by HG-HFFAs. In other words, these results suggested that the sympathetic excitement in T2DM-HFFA patients may give rise to functional and structural damage to the cardiac microvasculature, finally leading to sudden cardiac death. Further study of these findings may help uncover the pathological mechanism why strenuous exercise can give rise to angina or even sudden cardiac death.

However, in a study of rats, it was found that the degree in phosphorylation of Plin5 was not consistent with the hydrolysis of LDs in skeletal muscle cells [23]. Interestingly, Kuramoto et al. also proved that in fed condition, Plin $5^{-/-}$reduced the content of FFAs in the liver, but not in the heart and soleus muscle, whereas in fasted condition, Plin $5^{-1-}$ increased FFAs content in the liver and reduced it in the heart and soleus muscle. This discrepancy may prove again that the concrete roles of Plin5 depend on the species, the tissues, and eating or not.

The generation and removal of free radicals in the body are in equilibrium under normal conditions [69]. But in the pathological condition, the production of free radicals is increased, and the ability of antioxidation in body is decreased, resulting in lipid peroxidation, protein degeneration, and DNA damage [70]. The main marker of oxidative stress is excessive ROS, which is mainly produced from mitochondria. Studies have shown that endothelial cells supply their own energy mainly through glycolysis [71], but it has been reported that endothelial cells still can carry out large amounts of mitochondrial $\beta$-oxidation for energy supply under the stimulation of acute HFFAs [17]. Thus, we believe that mitochondrial ROS produced from $\beta$-oxidation cannot be ignored in the CMECs injury of T2DM-HFFA. Consistently, our studies revealed again that increased intracellular FFAs content led to excessive production of ROS. As well known, CPT-1 is the first speed-limiting enzyme in the process of mitochondrial fatty acid oxidation, and it can catalyze the long-chain fatty acids from the cytoplasm into the mitochondria $[72,73]$. In in vitro cell experiment, we found that the deletion and phosphorylation of Plin5 in CMECs increased mRNA expression of CPT-1 and raised intracellular level of ROS under the condition of HG-HFFAs. Consistently, de Barros Reis et al. reported that the increase in CPT-1 activity enhanced FFAs oxidation in islet cells [74]. Moreover, NAC intervention reduced the content of ROS, partly reversed the CMECs injury induced by the deletion and phosphorylation of Plin5. In line with our studies, many previous studies claimed that Plin5 knockout was able to increase the level of FFAs, enhance the $\beta$-oxidation of FFAs, and ultimately increase the production of $\operatorname{ROS}[14,31]$. In myocardial ischemia-reperfusion injury, it has been reported that Plin5 ablation can increase the content of ROS and malondialdehyde (MDA), but reduce the level of superoxide dismutase (SOD) [31]. Therefore, these results suggest that ROS overload derived from FFAs $\beta$-oxidation contributes to CMECs injury induced by the disruption of Plin 5 balance in T2DM-HFFA.

\section{Conclusion}

HFFAs can aggravate HG-induced cardiac microvascular injury; Plin5 in CMECs plays a bidirectionally role in FFAs metabolism via the states of nonphosphorylation and phosphorylation; the deletion or the phosphorylation of Plin5 will break the balance of FFAs metabolism, result in the excessive production of FFAs and the overload of ROS, and eventually aggravate the CMECs injury induced by T2DM-HFFA. This suggests that only appropriate expression and phosphorylation of Plin5 are beneficial to energy supply and fine regulation of FFAs metabolism in CMECs. In short, our study suggests that Plin 5 can guard the orderly metabolism of FFAs in CMECs in different states, and it can be explored as a new target for the prevention and treatment of microvascular complications in T2DM-HFFA. 


\begin{tabular}{ll} 
Abbreviations \\
HFFA: & Hyper-free fatty academia \\
T2DM: & Type 2 diabetes mellitus \\
Plin5: & Perilipin 5 \\
FFAs: & Free fatty acids \\
CMECs: & Cardiac microvascular endothelial cells \\
HG: & High glucose \\
HFFAs: & High FFAs \\
LDs: & Lipid droplets \\
NO: & Nitric oxide \\
ROS: & Reactive oxygen species \\
eNOS: & Endothelial nitric oxide synthase \\
NEFAs: & Nonesterified fatty acids \\
CMD: & Coronary microvascular diseases \\
TG: & Triglyceride \\
PKA: & Protein kinase A \\
PGC-1 $\alpha:$ & Peroxisome proliferator-activated receptor \\
& gamma coactivator $1-\alpha$ \\
RNS: & Reactive nitrogen species \\
STZ: & Streptozocin \\
CHOL: & Cholesterol \\
INS: & Insulin \\
ABS: & Acrylonitrile butadiene styrene \\
BSA: & Bovine serum albumin \\
LVEF: & Left ventricular ejection fraction \\
E/A: & Left ventricular early mitral diastolic wave/late \\
& mitral diastolic wave \\
PBS: & Phosphate-buffered saline \\
FBS: & Fetal bovine serum \\
vWF: & von Willebrand factor \\
ISO: & Isoprenaline \\
LSCM: & Laser confocal microscopy \\
ELISA: & Enzyme-linked immunosorbent assay \\
HBSS: & Hank's balanced salt solution \\
qRT-PCR: & Quantitative real-time polymerase chain \\
& reaction. \\
& \\
\hline
\end{tabular}

\section{Data Availability}

The data used to support the findings of this study are included within the article.

\section{Consent}

The consent to participate was obtained from the participants. Written informed consent for publication of their clinical details and/or clinical images was obtained from the patient. A copy of the consent form is available for review by the editor of this journal.

\section{Conflicts of Interest}

The authors declare that they have no competing interests.

\section{Authors' Contributions}

Yongjian Yang and Haifeng Pei drafted the manuscript. Jin $\mathrm{Du}$, Juanni Hou, and Juan Feng participated in the design of the study and performed the statistical analysis. Dachun
Yang, De Li, Hong Zhou, and Heng Zhao conceived the study and participated in its design and coordination and helped to draft the manuscript. All authors read and approved the final manuscript. Jin Du and Juanni Hou equally contributed to this work.

\section{Acknowledgments}

This work was supported by grants from the National Natural Science Foundation of China (Nos. 81970241, 81873477 and 81670419), the Science Fund for Distinguished Young Scholars of Sichuan Province (No. 2017JQ0012), and the China Postdoctoral Science Foundation (No. 2017M613429).

\section{Supplementary Materials}

Table 1: basal physiological parameters in all kinds of mice. Plin5: perilipin 5; BW: body weight; HW: heart weight; GLU: glucose; INS: insulin; TG: triglyceride; FFAs: free fatty acids; CHOL: total cholesterol. Presented values are mean \pm SEM, $n=8$-10/group. ${ }^{*} P<0.05,{ }^{* *} P<0.01$ vs. WT of the same genotypes. Supplementary Figure 1: identification of cardiac microvascular endothelial cells (CMECs) and T2DM-HFFA caused damage to CMECs. (A) Cell immunofluorescence staining. DAPI: $4^{\prime}$,6-diamidino-2-phenylindole (blue fluorescence); CD31: (green fluorescence); vWF: von Willebrand factor (red fluorescence). (B) The apoptosis rate measured by Annexin V-FITC/PI assay kit. (C) NO generation in CMECs measured by ELISA kit. T2DM-HFFA: type 2 diabetes mellitus with hyper-free fatty acidemia; ELISA: enzyme-linked immunosorbent assay. Data are expressed as mean \pm SEM, $n=6$-8/group. ${ }^{\theta} P<0.05,{ }^{\theta \theta} P<0.01$ vs. control. Supplementary Figure 2: identification of mouse genotypes. Polymerase chain reaction was used to identify WT and Plin $^{-1-}$ mice. Supplementary Figure 3: the impact of Plin5 knockdown on the injury of CMECs induced by HG-HFFAs. (A) The apoptosis rate measured by Annexin V-FITC/PI assay kit. (B) NO generation in CMECs measured by ELISA kit. HG-HFFAs, high glucose and high free fatty acids; Scra siRNA, scrambled siRNA; ELISA, enzyme-linked immunosorbent assay. Data are expressed as mean \pm SEM, $n=6-8$ / group. ${ }^{*} P<0.05$ vs. HG-HFFAs of Scra siRNA. Supplementary Figure 4: the effect of Plin5 knockout on eNOS in CMECs. (A) The activity of eNOS measured by eNOS Quantitation Kit. (B) The protein level of eNOS in CMECs determined by western blot. WT, wild type; eNOS, endothelial nitric oxide synthase; HG-HFFAs, high glucose and high free fatty acids. Data are expressed as mean \pm SEM, $n=6-8$ / group. ${ }^{* *} P<0.01$ vs. WT of normal; ${ }^{\# \#} P<0.01$ vs. Plin $5^{-/}$of normal; ${ }^{\&} P<0.05$ vs. WT of HG-HFFAs. Supplementary Figure 5: the effect of Plin5 phosphorylation on eNOS in CMECs. (A) The activity of eNOS measured by eNOS Quantitation Kit. (B) The protein level of eNOS in CMECs determined by western blot. Vel, vehicle; ISO, isoproterenol; eNOS, endothelial nitric oxide synthase; HG-HFFAs, high glucose and high free fatty acids. Data are expressed as mean \pm SEM, $n=6$-8/group. ${ }^{* *} P<0.01$ vs. Vel of normal; ${ }^{\# \#} P<0.01$ vs. ISO of normal; ${ }^{\circledR} P<0.05$ vs. Vel of HGHFFAs. Supplementary Figure 6: the effect of Plin5/p-Plin5 
on the mRNA expression of CPT-1 and ROS content in CMECs under the condition of HG-HFFAs. (A, B) The production of ROS in CMECs was measured by ELISA kit. (C, D) CPT-1 mRNA expression in CMECs measured by qRTPCR. CPT-1, carnitine palmitoyltransferase I; HG-HFFAs, high glucose and high free fatty acids; NAC, N-acetylcysteine; Vel, vehicle; ISO, isoproterenol; ELISA, enzymelinked immunosorbent assay; ROS, reactive oxygen species; qRT-PCR, quantitative real-time polymerase chain reaction. Presented values are mean \pm SEM, $n=6$-8/group. ${ }^{* *} P<$ 0.01 vs. WT + Vel; ${ }^{\# \#} P<0.01$ vs. Plin $5^{-1-}+$ Vel; ${ }^{\& \&} P<0.01$ vs. vehicle; ${ }^{@} P<0.01$ vs. ISO. (Supplementary Materials)

\section{References}

[1] S. Sharma, H. Singh, N. Ahmad, P. Mishra, and A. Tiwari, "The role of melatonin in diabetes: therapeutic implications," Archives of Endocrinology and Metabolism, vol. 59, no. 5, pp. 391-399, 2015.

[2] H. Zheng, J. Wu, Z. Jin, and L. J. Yan, "Potential biochemical mechanisms of lung injury in diabetes," Aging and Disease, vol. 8, no. 1, pp. 7-16, 2017.

[3] L. Yan, M. T. Xu, L. Yuan et al., "Prevalence of dyslipidemia and its control in type 2 diabetes: a multicenter study in endocrinology clinics of China," Journal of Clinical Lipidology, vol. 10, no. 1, pp. 150-160, 2016.

[4] G. Boden, "Obesity and free fatty acids," Endocrinology and Metabolism Clinics of North America, vol. 37, no. 3, pp. 635646, 2008.

[5] B. M. Egan, E. L. Greene, and T. L. Goodfriend, "Nonesterified fatty acids in blood pressure control and cardiovascular complications," Current Hypertension Reports, vol. 3, no. 2, pp. 107-116, 2001.

[6] Y. Lee, H. Hirose, M. Ohneda, J. H. Johnson, J. D. McGarry, and R. H. Unger, "Beta-cell lipotoxicity in the pathogenesis of non-insulin-dependent diabetes mellitus of obese rats: impairment in adipocyte-beta-cell relationships," Proceedings of the National Academy of Sciences of the United States of America, vol. 91, no. 23, pp. 10878-10882, 1994.

[7] M. Nyberg, L. Gliemann, and Y. Hellsten, "Vascular function in health, hypertension, and diabetes: effect of physical activity on skeletal muscle microcirculation," Scandinavian Journal of Medicine \& Science in Sports, vol. 25, Supplement 4, pp. 60-73, 2015.

[8] M. Joshi, S. R. Kotha, S. Malireddy et al., "Conundrum of pathogenesis of diabetic cardiomyopathy: role of vascular endothelial dysfunction, reactive oxygen species, and mitochondria," Molecular and Cellular Biochemistry, vol. 386, no. 1-2, pp. 233-249, 2014.

[9] R. S. Rosenson, P. Fioretto, and P. M. Dodson, "Does microvascular disease predict macrovascular events in type 2 diabetes?," Atherosclerosis, vol. 218, no. 1, pp. 13-18, 2011.

[10] D. Antoniucci, R. Valenti, A. Migliorini et al., "Direct infarct artery stenting without predilation and no-reflow in patients with acute myocardial infarction," American Heart Journal, vol. 142, no. 4, pp. 684-690, 2001.

[11] J. K. Zehmer, Y. Huang, G. Peng, J. Pu, R. G. W. Anderson, and P. Liu, "A role for lipid droplets in inter-membrane lipid traffic,” Proteomics, vol. 9, no. 4, pp. 914-921, 2009.

[12] K. T. Dalen, T. Dahl, E. Holter et al., "Lsdp5 is a pat protein specifically expressed in fatty acid oxidizing tissues," Biochi- mica et Biophysica Acta (BBA) - Molecular and Cell Biology of Lipids, vol. 1771, no. 2, pp. 210-227, 2007.

[13] H. Wang, U. Sreenivasan, H. Hu et al., "Perilipin 5, a lipid droplet-associated protein, provides physical and metabolic linkage to mitochondria," Journal of Lipid Research, vol. 52, no. 12, pp. 2159-2168, 2011.

[14] K. Kuramoto, T. Okamura, T. Yamaguchi et al., "Perilipin 5, a lipid droplet-binding protein, protects heart from oxidative burden by sequestering fatty acid from excessive oxidation," Journal of Biological Chemistry, vol. 287, no. 28, pp. 2385223863, 2012.

[15] R. R. Mason, R. Mokhtar, M. Matzaris et al., "Plin5 deletion remodels intracellular lipid composition and causes insulin resistance in muscle," Molecular Metabolism, vol. 3, no. 6, pp. 652-663, 2014.

[16] C. Wang, Y. Zhao, X. Gao et al., "Perilipin 5 improves hepatic lipotoxicity by inhibiting lipolysis," Hepatology, vol. 61, no. 3, pp. 870-882, 2015.

[17] A. Kuo, M. Y. Lee, and W. C. Sessa, "Lipid droplet biogenesis and function in the endothelium," Circulation Research, vol. 120, no. 8, pp. 1289-1297, 2017.

[18] P.-L. Zhou, M. Li, X.-W. Han et al., "Perilipin 5 deficiency promotes atherosclerosis progression through accelerating inflammation, apoptosis, and oxidative stress," Journal of Cellular Biochemistry, vol. 120, no. 11, pp. 19107-19123, 2019.

[19] H. Wang, U. Sreenivasan, D. W. Gong et al., "Cardiomyocytespecific perilipin 5 overexpression leads to myocardial steatosis and modest cardiac dysfunction," Journal of Lipid Research, vol. 54, no. 4, pp. 953-965, 2013.

[20] N. M. Pollak, M. Schweiger, D. Jaeger et al., "Cardiac-specific overexpression of perilipin 5 provokes severe cardiac steatosis via the formation of a lipolytic barrier," Journal of Lipid Research, vol. 54, no. 4, pp. 1092-1102, 2013.

[21] R. R. Mason and M. J. Watt, "Unraveling the roles of plin5: linking cell biology to physiology," Trends in Endocrinology and Metabolism, vol. 26, no. 3, pp. 144-152, 2015.

[22] N. M. Pollak, D. Jaeger, S. Kolleritsch et al., "The interplay of protein kinase A and perilipin 5 regulates cardiac lipolysis," Journal of Biological Chemistry, vol. 290, no. 3, pp. 12951306, 2015.

[23] R. E. K. Macpherson, R. Vandenboom, B. D. Roy, and S. J. Peters, "Skeletal muscle plin3 and plin5 are serine phosphorylated at rest and following lipolysis during adrenergic or contractile stimulation," Physiological Reports, vol. 1, no. 4, article e00084, 2013.

[24] V. I. Gallardo-Montejano, G. Saxena, C. M. Kusminski et al., "Nuclear perilipin 5 integrates lipid droplet lipolysis with PGC- $1 \alpha /$ SIRT1-dependent transcriptional regulation of mitochondrial function," Nature Communications, vol. 7, no. 1, article 12723, 2016.

[25] X. Gao, Y. Nan, Y. Zhao et al., "Atorvastatin reduces lipid accumulation in the liver by activating protein kinase Amediated phosphorylation of perilipin 5," Biochimica et Biophysica Acta (BBA) - Molecular and Cell Biology of Lipids, vol. 1862, no. 12, pp. 1512-1519, 2017.

[26] H. Pei, Q. Yu, Q. Xue et al., "Notch1 cardioprotection in myocardial ischemia/reperfusion involves reduction of oxidative/nitrative stress," Basic Research in Cardiology, vol. 108, no. 5, p. 373, 2013.

[27] A. Grindel, B. Guggenberger, L. Eichberger et al., "Oxidative stress, DNA damage and DNA repair in female patients with 
diabetes mellitus type 2," PLoS One, vol. 11, no. 9, article e0162082, 2016.

[28] H. P. Indo, H. C. Yen, I. Nakanishi et al., "A mitochondrial superoxide theory for oxidative stress diseases and aging," Journal of Clinical Biochemistry and Nutrition, vol. 56, no. 1, pp. 1-7, 2015.

[29] M. F. Cury-Boaventura and R. Curi, "Regulation of reactive oxygen species (ROS) production by $\mathrm{C}_{18}$ fatty acids in Jurkat and Raji cells," Clinical Science, vol. 108, no. 3, pp. 245-253, 2005.

[30] J. Kim, Y. Wei, and J. R. Sowers, "Role of mitochondrial dysfunction in insulin resistance," Circulation Research, vol. 102, no. 4, pp. 401-414, 2008.

[31] P. Zheng, Z. Xie, Y. Yuan et al., "Plin5 alleviates myocardial ischaemia/reperfusion injury by reducing oxidative stress through inhibiting the lipolysis of lipid droplets," Scientific Reports, vol. 7, no. 1, article 42574, 2017.

[32] H. Lee and Y. Lim, "Tocotrienol-rich fraction supplementation reduces hyperglycemia-induced skeletal muscle damage through regulation of insulin signaling and oxidative stress in type 2 diabetic mice," The Journal of Nutritional Biochemistry, vol. 57, pp. 77-85, 2018.

[33] H. T. Pham, W. Huang, C. Han et al., "Effects of Averrhoa carambola L. (Oxalidaceae) juice mediated on hyperglycemia, hyperlipidemia, and its influence on regulatory protein expression in the injured kidneys of streptozotocin-induced diabetic mice," American Journal of Translational Research, vol. 9, no. 1, pp. 36-49, 2017.

[34] Y. T. Zhou, P. Grayburn, A. Karim et al., "Lipotoxic heart disease in obese rats: implications for human obesity," Proceedings of the National Academy of Sciences of the United States of America, vol. 97, no. 4, pp. 1784-1789, 2000.

[35] J. Li, J. Gong, X. Li, L. Shen, Y. Xie, and R. Zhang, "MicroRNA34a promotes CMECS apoptosis and upregulate inflammatory cytokines, thus worsening CMECS damage and inhibiting angiogenesis by negatively targeting the Notch signaling pathway," Journal of Cellular Biochemistry, vol. 120, no. 2, pp. 1598-1609, 2019.

[36] A. Pavlosky, A. Lau, Y. Su et al., "RIPK3-mediated necroptosis regulates cardiac allograft rejection," American Journal of Transplantation, vol. 14, no. 8, pp. 1778-1790, 2014.

[37] Y. Xue, T. Guo, L. Zou et al., "Evodiamine attenuates $\mathrm{P}_{2} \mathrm{X}_{7^{-}}$ mediated inflammatory injury of human umbilical vein endothelial cells exposed to high free fatty acids," Oxidative Medicine and Cellular Longevity, vol. 2018, Article ID 5082817, 10 pages, 2018.

[38] X. Li, J. Hou, J. du et al., "Potential protective mechanism in the cardiac microvascular injury," Hypertension, vol. 72, no. 1, pp. 116-127, 2018.

[39] H. F. Pei, J. N. Hou, F. P. Wei et al., "Melatonin attenuates postmyocardial infarction injury via increasing Tom70 expression," Journal of Pineal Research, vol. 62, no. 1, 2017.

[40] Q. Xue, H. Pei, Q. Liu et al., "MICU1 protects against myocardial ischemia/reperfusion injury and its control by the importer receptor Tom70," Cell Death \& Disease, vol. 8, no. 7, article e2923, 2017.

[41] P. Zimmet, K. G. Alberti, D. J. Magliano, and P. H. Bennett, "Diabetes mellitus statistics on prevalence and mortality: facts and fallacies," Nature Reviews Endocrinology, vol. 12, no. 10, pp. 616-622, 2016.
[42] T. Mazzone, "Intensive glucose lowering and cardiovascular disease prevention in diabetes: reconciling the recent clinical trial data," Circulation, vol. 122, no. 21, pp. 2201-2211, 2010.

[43] M. P. de Boer, R. I. Meijer, N. J. Wijnstok et al., "Microvascular dysfunction: a potential mechanism in the pathogenesis of obesity-associated insulin resistance and hypertension," Microcirculation, vol. 19, no. 1, pp. 5-18, 2012.

[44] A. Gupta, A. K. Gupta, and T. P. Singh, "Occurrence of complications in newly diagnosed type 2 diabetes patients: a hospital based study," Journal of the Indian Medical Association, vol. 111, no. 4, pp. 245-247, 2013.

[45] Y. Liu, Y. Ma, R. Wang et al., "Advanced glycation end products accelerate ischemia/reperfusion injury through receptor of advanced end product/nitrative thioredoxin inactivation in cardiac microvascular endothelial cells," Antioxidants \& Redox Signaling, vol. 15, no. 7, pp. 1769-1778, 2011.

[46] Z. Yin, L. Fan, L. Wei et al., "FTY720 protects cardiac microvessels of diabetes: a critical role of S1P1/3 in diabetic heart disease," PLoS One, vol. 7, no. 8, article e42900, 2012.

[47] M. Laakso, "Heart in diabetes: a microvascular disease," Diabetes Care, vol. 34, Supplement 2, pp. S145-S149, 2011.

[48] A. Virdis, "Endothelial dysfunction in obesity: role of inflammation," High Blood Pressure \& Cardiovascular Prevention, vol. 23, no. 2, pp. 83-85, 2016.

[49] D. Gao, C. Pararasa, C. R. Dunston, C. J. Bailey, and H. R. Griffiths, "Palmitate promotes monocyte atherogenicity via de novo ceramide synthesis," Free Radical Biology \& Medicine, vol. 53, no. 4, pp. 796-806, 2012.

[50] J. R. Durrant, D. R. Seals, M. L. Connell et al., "Voluntary wheel running restores endothelial function in conduit arteries of old mice: direct evidence for reduced oxidative stress, increased superoxide dismutase activity and down-regulation of NADPH oxidase," The Journal of Physiology, vol. 587, no. 13, pp. 3271-3285, 2009.

[51] S. Wang, A. Ma, S. Song, Q. Quan, X. Zhao, and X. Zheng, "Fasting serum free fatty acid composition, waist/hip ratio and insulin activity in essential hypertensive patients," Hypertension Research, vol. 31, no. 4, pp. 623-632, 2008.

[52] S. Pilz, H. Scharnagl, B. Tiran et al., "Free fatty acids are independently associated with all-cause and cardiovascular mortality in subjects with coronary artery disease," The Journal of Clinical Endocrinology and Metabolism, vol. 91, no. 7, pp. 2542-2547, 2006.

[53] M. Pirro, P. Mauriège, A. Tchernof et al., "Plasma free fatty acid levels and the risk of ischemic heart disease in men: prospective results from the Québec cardiovascular study," Atherosclerosis, vol. 160, no. 2, pp. 377-384, 2002.

[54] S. X. Guo, Y. Z. Yan, L. T. Mu et al., “Association of serum free fatty acids with hypertension and insulin resistance among rural Uyghur adults in far western China," International Journal of Environmental Research and Public Health, vol. 12, no. 6, pp. 6582-6590, 2015.

[55] O. Brouwers, P. M. Niessen, G. Haenen et al., "Hyperglycaemia-induced impairment of endothelium-dependent vasorelaxation in rat mesenteric arteries is mediated by intracellular methylglyoxal levels in a pathway dependent on oxidative stress," Diabetologia, vol. 53, no. 5, pp. 989-1000, 2010.

[56] A. Ghosh, L. Gao, A. Thakur, P. M. Siu, and C. W. K. Lai, "Role of free fatty acids in endothelial dysfunction," Journal of Biomedical Science, vol. 24, no. 1, p. 50, 2017. 
[57] H. Li, H. Li, Y. Bao, X. Zhang, and Y. Yu, "Free fatty acids induce endothelial dysfunction and activate protein kinase $\mathrm{C}$ and nuclear factor- $\kappa \mathrm{B}$ pathway in rat aorta," International Journal of Cardiology, vol. 152, no. 2, pp. 218-224, 2011.

[58] S. D. Kohlwein, M. Veenhuis, and I. J. van der Klei, "Lipid droplets and peroxisomes: key players in cellular lipid homeostasisora matter of fat-store 'em up or burn 'em down," Genetics, vol. 193, no. 1, pp. 1-50, 2013.

[59] H. Wang, M. Bell, U. Sreenevasan et al., "Unique regulation of adipose triglyceride lipase (ATGL) by perilipin 5, a lipid droplet-associated protein," Journal of Biological Chemistry, vol. 286, no. 18, pp. 15707-15715, 2011.

[60] C. Bindesboll, O. Berg, B. Arntsen, H. I. Nebb, and K. T. Dalen, "Fatty acids regulate perilipin5 in muscle by activating PPARס," Journal of Lipid Research, vol. 54, no. 7, pp. 19491963, 2013.

[61] E. Grasselli, A. Voci, C. Pesce et al., "Pat protein mRNA expression in primary rat hepatocytes: effects of exposure to fatty acids," International Journal of Molecular Medicine, vol. 25 , no. 4 , pp. 505-512, 2010.

[62] A. M. Hall, E. M. Brunt, Z. Chen et al., "Dynamic and differential regulation of proteins that coat lipid droplets in fatty liver dystrophic mice," Journal of Lipid Research, vol. 51, no. 3, pp. 554-563, 2010.

[63] H. Li, Y. Song, L. J. Zhang et al., "LSDP5 enhances triglyceride storage in hepatocytes by influencing lipolysis and fatty acid $\beta$ oxidation of lipid droplets," PLoS One, vol. 7, no. 6, article e36712, 2012.

[64] R. Rinnankoski-Tuikka, J. J. Hulmi, S. Torvinen et al., "Lipid droplet-associated proteins in high-fat fed mice with the effects of voluntary running and diet change," Metabolism: Clinical and Experimental, vol. 63, no. 8, pp. 1031-1040, 2014.

[65] P. M. Badin, I. K. Vila, K. Louche et al., "High-fat dietmediated lipotoxicity and insulin resistance is related to impaired lipase expression in mouse skeletal muscle," Endocrinology, vol. 154, no. 4, pp. 1444-1453, 2013.

[66] K. Kuramoto, F. Sakai, N. Yoshinori et al., "Deficiency of a lipid droplet protein, perilipin 5, suppresses myocardial lipid accumulation, thereby preventing type 1 diabetes-induced heart malfunction," Molecular and Cellular Biology, vol. 34, no. 14, pp. 2721-2731, 2014.

[67] S. H. Ibrahim, R. Kohli, and G. J. Gores, "Mechanisms of lipotoxicity in NAFLD and clinical implications," Journal of Pediatric Gastroenterology and Nutrition, vol. 53, no. 2, pp. 131-140, 2011.

[68] S. Viswanadha and C. Londos, "Determination of lipolysis in isolated primary adipocytes," Methods in Molecular Biology, vol. 456, pp. 299-306, 2008.

[69] B. D'Autreaux and M. B. Toledano, "ROS as signalling molecules: mechanisms that generate specificity in ROS homeostasis," Nature Reviews Molecular Cell Biology, vol. 8, no. 10, pp. 813-824, 2007.

[70] G. Bellomo, "Cell damage by oxygen free radicals," Cytotechnology, vol. 5, Supplement 1, pp. 71-73, 1991.

[71] O. Culic, M. L. Gruwel, and J. Schrader, "Energy turnover of vascular endothelial cells," American Journal of PhysiologyCell Physiology, vol. 273, no. 1, pp. C205-C213, 1997.

[72] J. Kerner and C. Hoppel, "Fatty acid import into mitochondria," Biochimica et Biophysica Acta (BBA) - Molecular and Cell Biology of Lipids, vol. 1486, no. 1, pp. 1-17, 2000.
[73] J. D. McGarry and N. F. Brown, "The mitochondrial carnitine palmitoyltransferase system - from concept to molecular analysis," European Journal of Biochemistry, vol. 244, no. 1, pp. 1-14, 1997.

[74] M. A. de Barros Reis, V. C. Arantes, D. A. Cunha et al., "Increased L-CPT-1 activity and altered gene expression in pancreatic islets of malnourished adult rats: a possible relationship between elevated free fatty acid levels and impaired insulin secretion," The Journal of Nutritional Biochemistry, vol. 19 , no. 2 , pp. 85-90, 2008. 


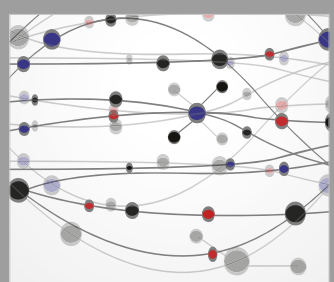

The Scientific World Journal
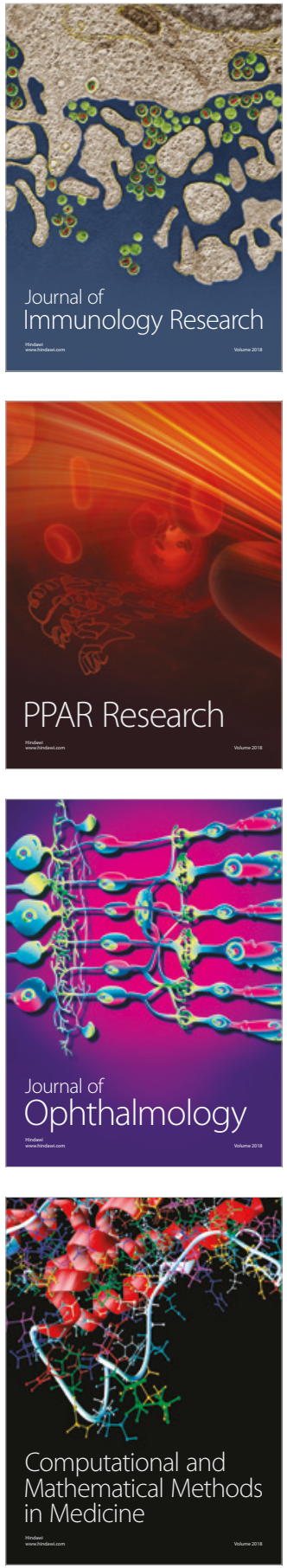

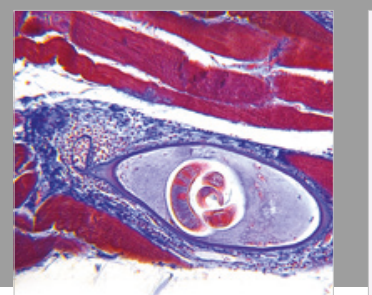

Gastroenterology Research and Practice

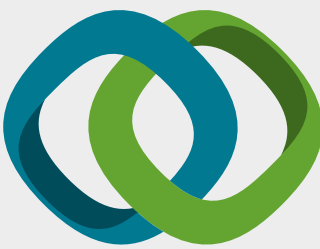

\section{Hindawi}

Submit your manuscripts at

www.hindawi.com
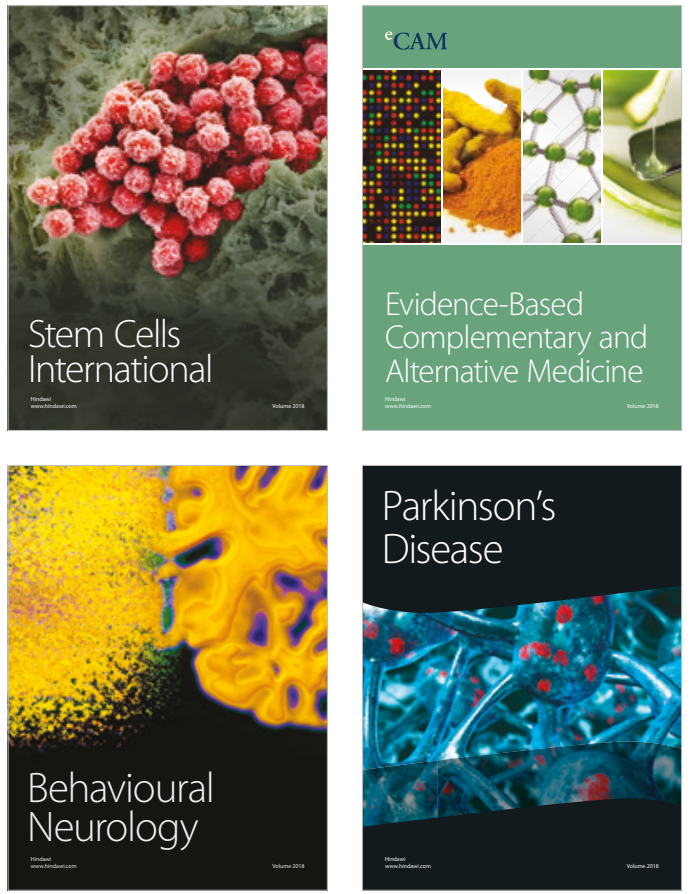

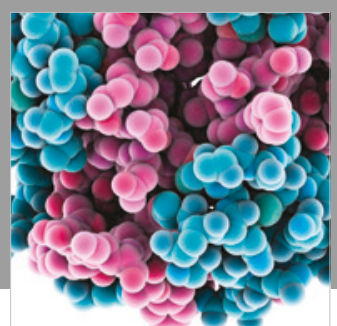

ournal of

Diabetes Research

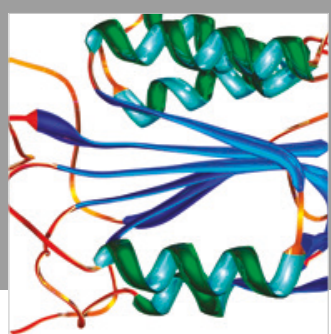

Disease Markers
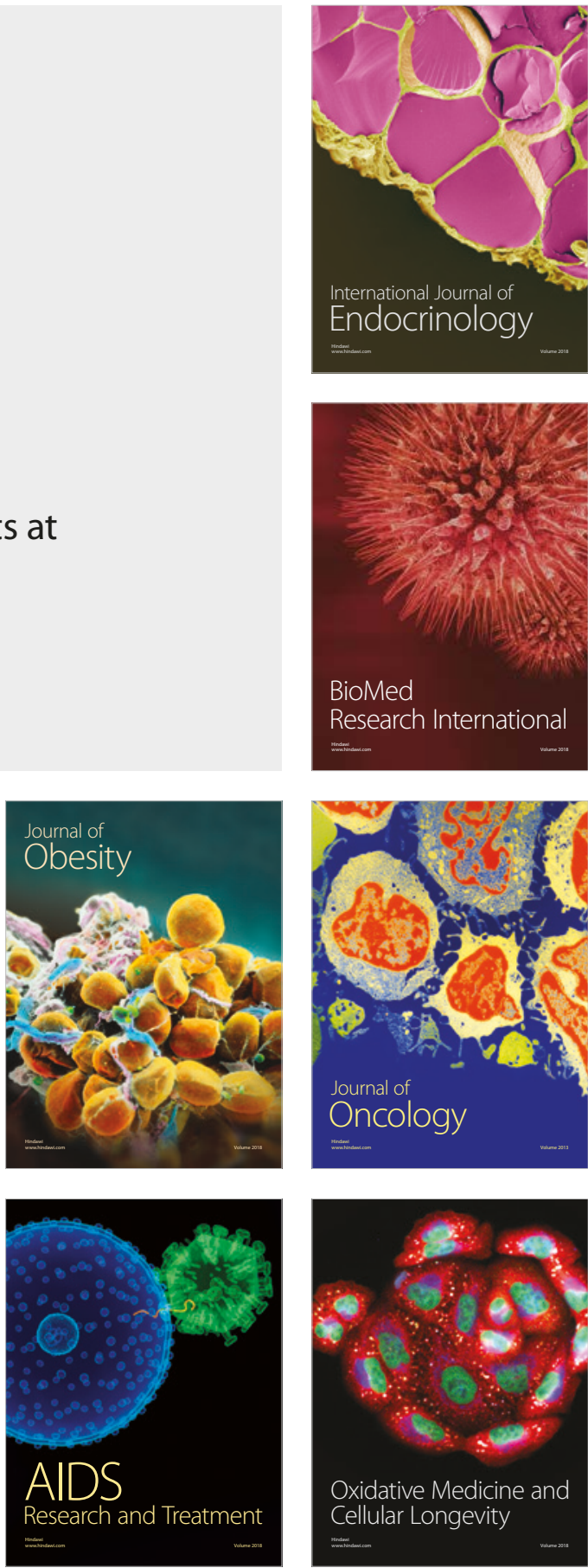